\title{
Energy-Limited Time-Varying Formation Control for Second-Order Multiagent Systems
}

\author{
Wanzhen Quan, ${ }^{1}$ Yulong Zhao, ${ }^{2}$ Le Wang $\mathbb{D}^{1},{ }^{1}$ and Xiaogang Yang $\mathbb{D}^{1}$ \\ ${ }^{1}$ High-Tech Institute of Xi'an, Xi'an 710025, China \\ ${ }^{2}$ College of Information and Communication, University of National Defense Technology, Wuhan 430010, China
}

Correspondence should be addressed to Xiaogang Yang; doctoryxg@163.com

Received 20 August 2020; Revised 12 September 2020; Accepted 8 October 2020; Published 12 November 2020

Academic Editor: Ning Cai

Copyright ( $) 2020$ Wanzhen Quan et al. This is an open access article distributed under the Creative Commons Attribution License, which permits unrestricted use, distribution, and reproduction in any medium, provided the original work is properly cited.

\begin{abstract}
The energy-limited time-varying formation (ETVF) control problem of second-order multiagent systems (MAS) is addressed for both leaderless and leader-following communication topologies in this paper. Different from the previous results, the joint consideration of energy limitation and formation design is more challenging and practical. First, an ETVF control protocol is presented, and the total energy supply is pregiven and limited, which is more common in practical applications. Then, by an orthogonal transformation, the formation control problem is converted into the consensus stabilization problem for second-order leaderless MAS, where sufficient conditions for the ETVF are derived by joint design of control gains and the total energy. At the same time, the explicit formula that forms the formation center function is obtained to depict the macroscopic movement of the multiagent system as a whole. Moreover, the proposed method is also extended to the leader-following communication structure. Finally, two examples are given to verify the effectiveness of our theoretical results.
\end{abstract}

\section{Introduction}

Recently, the topic of cooperative control of MAS has been paid increasing attention due to its wide application in many fields, such as sensor network synchronization [1-3], formation control [4-7], flocking $[8,9]$, and so on. Cooperative control of MAS originated from bioscience, where many researchers aroused great interest in birds, bees, and fishes in nature. Many scholars found that the above cooperative behavior among agents is based on the swarm consensus [10-17], and all agents could perform tasks that cannot be achieved by a single one as a formation structure. The formation control, as a fundamental and key issue of cooperative control, refers to a team of interacting agents to perform complex tasks together in a certain geometric structure, which is applied to many fields, such as networking system of satellite clusters, movements of mobile robotics $[18,19]$, cooperative attack of multiple missiles [20], and unmanned aerial vehicle formations $[21,22]$.
It is well known that the formation can be divided into the time-invariant and time-varying formation according to variation of formation structures. Fabris et al. [23] investigated the time-invariant formation tracking problem for second-order MAS, and a special potential function was designed to obtain a specified geometric structure, where the position and velocity of second-order MAS followed their expected values. Based on the virtual structure and consensus method, a formation control strategy was proposed in [24] to analyze nonholonomic intelligent vehicles. However, these methods are only suitable for the fixed formation structure. In practice, the application scenario is dynamic, and the formation of MAS needs to vary over time, such as obstacle avoidance. Accordingly, it is necessary to investigate the problem of time-varying formation control. In [25], an observer-based protocol was proposed to obtain the distributed time-varying group formation under the directed communication topology for MAS. Dong et al. [26] focused on the time-varying formation tracking problem with switching communication topologies for second-order 
MAS, and the effectiveness of the proposed theoretical results were proved by the multiquadrotor unmanned aerial vehicle system.

Besides, there are two types of formation, called leaderless formation and leader-following formation, depending on whether or not the communication topology among agents has a specified leader. A finite-time cooperative controller was proposed for multiple leaderless nonholonomic mobile robots via the finite-time control technique and the model features [27]. Jia et al. [28] investigated the three-dimensional leaderless flocking problem and proposed a simplified distributed control algorithm, which was verified by the LaSalle-Krasovskii invariance principle. Furthermore, the leader-following formation aroused much attention in [29-33], where followers tracked the leader and maintained a specified formation with it. Considering the condition of uncertain factors and time delay, the leader-follower collaborative formation was achieved for the multi-unmanned underwater vehicle formation in [32]. The time-varying protocol of second-order leader-following MAS was obtained in [33] through solving an algebraic Riccati equation.

Despite the numerous results mentioned above, there is still much uncompleted interesting work when considering the energy limitation problem in practical engineering applications. For example, when unmanned aerial vehicles execute some flight tasks of agricultural seeding, forest fire prevention, and cooperative reconnaissance, their fuel or battery power is limited, and it is difficult to further recharge energy during the flight. Therefore, it is necessary not only to achieve formation control but also to consider limited energy. Under the circumstance, the energy-limited formation control problem is converted to the optimal/suboptimal problem to guarantee some performance index. However, the time-varying formation design for second-order MAS was addressed in $[34,35]$, where the problem of energy limitation was not considered. Note that the optimizing consensus was investigated in $[36,37]$. An optimal consensus control for multiagent linear systems was investigated in the cases of the all-to-all and general communication [38], and the result illustrated that the proposed controller made the state of the closed-loop system converge exponentially. In [36-38], optimizing control was just the optimization of consensus, but the formation control problem of energy limitation was not taken into consideration. In practice, the total energy of MAS is usually constrained, and it is significant to study the formation control problem of energy limitation for MAS, which is an important basis for our investigation of this paper.

Motivated by the aforementioned facts and challenges, the problems of time-varying formation for MAS with limited energy in leaderless and leader-following cases are studied in this paper, respectively. First, an ETVF control protocol for second-order MAS is proposed for the leaderless communication topology. In addition, through orthogonal transformation, the dynamics of the multiagent system can be resolved into two independent parts: the consistency component and the inconsistent component. The former determines the whole macroscopic motion, and the microscopic motion is obtained by the latter. Second, sufficient conditions with analytic solutions of control gains and a formation center function are obtained in the leaderless case, in which the formation center function refers to macroscopic movements of the whole multiagents. Third, the research on the leaderless case is extended to the leaderfollowing one. It is worth mentioning that a two-step method to transform the leader-follower into the leaderless framework is proposed.

The main contributions of this paper are threefold. Firstly, the problem of limited energy supply is taken into consideration based on practical engineering, where a joint design strategy combining time-varying formation control and energy constraints is investigated. In this case, the desired formation structure not only must be formed but also the practical energy consumption of the time-varying formation is less than the given energy, where the practical energy is a performance index function composed of the quadratic form of the control protocol. However, the formation control problem in [32-34] did not consider energy limitation. Secondly, in the process of solving the control gain, the numerical solution solved by Matlab's LMI Toolbox is replaced by a feasible analytical solution, so it is not needed to verify the feasibility of the solution. In [32-34], the control gains were obtained by the LMI Toolbox instead of the analytical solution. Thirdly, the critical contribution is that a two-step transformation method is presented so as to transform the whole Laplacian matrix into the dimensionlowering diagonal matrix; in this case, the leaderless and leader-following communication topologies are unified into the identical multiagent system framework.

This paper is organized as follows. The communication topology modeling and the problem description are introduced in Section 2. In Section 3, the stability of ETVF for second-order MAS is analyzed in the leaderless case, and the formation center function is determined. Section 4 extends the ETVF for the leaderless case to the leader-following one. Section 5 verifies the theoretical results by two numerical simulation examples. A brief conclusion is drawn in Section 6.

Notations. Let the symbol $\mathbb{R}$ denote a real constant. The matrix $I_{2}$ is an identity matrix of dimension 2. $1_{N}$ stands for the $N$-dimensional column vector with all elements being 1 . 0 represents the zero matrix or zero vector with all elements equal to 0 . Let the symbol $\otimes$ denote the Kronecker product.

\section{Problem Description}

2.1. Preliminaries: Modeling Communication Topology. For the second-order MAS with $N$ homogeneous agents, the communication topology among them is described by an undirected weighted graph $G=(V(G), E(G))$, where $V(G)=\left\{v_{1}, v_{2}, \ldots, v_{N}\right\}$ denotes the set of nodes, $E(G) \subseteq V(G) \times V(G)$ is the set of edges with $E(G)=\left\{e_{i j}=\left(v_{i}, v_{j}\right)\right\}$ being a communication link from the $i$-th agent to the $j^{\text {th }}$ agent, and edge $\left(v_{i}, v_{i}\right)$ is called the selfloop of vertex $v_{i}$. The self-loops of topology in this paper are not considered, and we assume that the topology has no self- 
loops. The index of the neighbor set of agent $i$ is denoted as $N_{i}=\left\{v_{j}:\left(v_{j}, v_{i}\right) \in E(G)\right\}$, and the weight adjacency matrix is defined as $W(G)=\left[w_{i j}\right] \in \mathbb{R}^{N \times N}$, where the weight $w_{i j}>0$ if $\left(v_{j}, v_{i}\right) \in E(G)$, and $w_{i j}=0$ otherwise. Let the degree matrix be $D(G)=\operatorname{diag}\left\{d_{1}, d_{2}, \ldots, d_{N}\right\}$, where the indegree matrix of agent $i$ is defined by $d_{i}=\sum_{j=1}^{N} w_{i j}$. The Laplacian matrix $L$ is defined as $L=D(G)-W(G)$. More details about the graph theory can be found in [39].

2.2. Designing Energy-Limited Formation Control Protocol. Consider a group of second-order homogeneous agents. The dynamics of each agent is modeled by

$$
\left\{\begin{array}{l}
\dot{x}_{i}(t)=v_{i}(t), \\
\dot{v}_{i}(t)=u_{i}(t),
\end{array}\right.
$$$$
\left\{\begin{array}{l}
u_{i}(t)=\sum_{j \in N_{i}} w_{i j}\left(k_{1}\left(x_{j}(t)-f_{j x}(t)-x_{i}(t)+f_{i x}(t)\right)+k_{2}\left(v_{j}(t)-f_{j v}(t)-v_{i}(t)+f_{i v}(t)\right)\right), \\
J_{u}=\sum_{i=1}^{N} \int_{0}^{+\infty} \kappa u_{i}^{2}(t) \mathrm{d} t,
\end{array}\right.
$$

where $i=1,2, \ldots, N, x_{i}(t) \in \mathbb{R}$ and $v_{i}(t) \in \mathbb{R}$ are the position and the velocity terms of the $i$-th agent, respectively, and $u_{i}(t) \in \mathbb{R}$ is the control input. The desired formation is designed by the time-varying formation function $f(t)=\left[f_{1}^{T}(t), f_{2}^{T}(t), \ldots, f_{N}^{T}(t)\right]^{T}$, where $f_{i}(t)=\left[f_{i x}(t)\right.$, $\left.f_{i v}(t)\right]^{T}$ is piecewise continuous differentiable with $f_{i x}(t)$ representing the position term and $f_{i v}(t)$ denotes the velocity term.

For multiagent system (1), an energy-limited formation control protocol is put forward as follows: where $i=1,2, \ldots, N$ and $\kappa$ is a positive constant (it is a weight coefficient for performance index function of system (1), and we can effectively inhibit the amplitude growth of the control quantity $u(t)$ by increasing $\kappa)$. $k_{1}$ and $k_{2}$ are control gains with $k_{1}, k_{2}>0$, and $J_{u}$ is an energy index function, which refers to the control energy consumption. Let $J_{u}^{*}$ be the total energy supply of second-order MAS; then, the definition of the energy-limited formation for secondorder MAS is given below.

Definition 1. For any given $J_{u}^{*}>0$ and nonidentical initial states $x_{i}(0)$ and $v_{i}(0)(i=1,2, \ldots, N)$, multiagent system (1) with formation control protocol (2) is said to be energylimited formation achievable if there exist control gains $k_{1}$ and $k_{2}$ such that $\lim _{t \longrightarrow+\infty}\left(x_{i}(t)-f_{i x}(t)-c_{x}(t)\right)=0$, $\lim _{t \rightarrow+\infty}\left(v_{i}(t)-f_{i v}(t)-c_{v}(t)\right)=0$, and $J_{u} \leq J_{u}^{*}$, where $c_{x}(t)$ and $c_{v}(t)$ correspond to the position and velocity terms of the formation center function $c(t)=\left[c_{x}(t), c_{v}(t)\right]^{T}$, respectively.

The fundamental purpose of this paper lies in the following two major aspects: (i) how to design control gains $k_{1}$ and $k_{2}$ such that multiagent system (1) with formation control protocol (2) can achieve the desired formation in which the total energy supply is limited and (ii) how to obtain the explicit formula of the formation center function.

Remark 1. It is important to emphasize that controller (2) looks so complicated, which shows the essence of the state feedback control. Firstly, controller (2) about $u(t)$ is the distributed consensus control protocol, which mainly consists of two parts, one is $x_{j}(t)-f_{j x}(t)-x_{i}(t)+f_{i x}(t)$ denoting the tracking error of position, and another is the tracking error of velocity $v_{j}(t)-f_{j v}(t)-v_{i}(t)+f_{i v}(t)$. Secondly, controller (2) about $J_{u}$ refers to the cost function that is the integral of $u(t)$ with respect to time $t$, which reflects the practical energy consumption for the whole system. The goal of consensus protocol (2) is to guarantee that $\quad x_{j}(t)-f_{j x}(t)-x_{i}(t)+f_{i x}(t) \longrightarrow 0 \quad$ and $v_{j}(t)-f_{j v}(t)-v_{i}(t)+f_{i v}(t) \longrightarrow 0$ as $t \longrightarrow \infty$; at the same time, the practical energy consumption is less than the total energy supply budget. And it is important to emphasize that controller (2) looks so complicated, but the states are knowable and easy to implement.

Remark 2. The formation control protocol (2) has two important characteristics. On the one hand, it contains an energy index function which is significant for practical engineering applications due to limited resources, such as fuel supply and battery power in practical engineering applications. Under this condition, it is crucial to propose a new idea that joins design between control gains $k_{1}, k_{2}$ and energy constraints. In other words, the control gains $k_{1}$ and $k_{2}$ are designed to maintain the desired formation and ensure that energy consumption of second-order MAS is within its total energy supply budget. On the other hand, the problem of the time-varying formation control is given, and a time-varying formation function is required to introduce into the control protocol (2), which makes the ETVF control problem for second-order MAS more challenging and practical than time-invariant ones, such as avoiding dynamic obstacles in time. And one can find that the time-varying formation not only forms a certain structure but also moves in a desired geometric formation according to the requirement of the realistic environments. 


\section{ETVF Control for the Leaderless Case}

This section presents sufficient conditions for multiagent system (1) with formation control protocol (2) to achieve the ETVF. Then, an explicit formula of the formation center function is presented.
Let $\delta_{i x}(t)=x_{i}(t)-f_{i x}(t)$ and $\delta_{i v}(t)=v_{i}(t)-f_{i v}(t)$; then, the dynamics of multiagent system (1) with formation control protocol (2) can be depicted as

$$
\left\{\begin{array}{l}
\dot{\delta}_{i x}(t)=\delta_{i v}(t)+f_{i v}(t)-\dot{f}_{i x}(t), \\
\dot{\delta}_{i v}(t)=k_{1} \sum_{j \in N_{i}} w_{i j}\left(\delta_{j x}(t)-\delta_{i x}(t)\right)+k_{2} \sum_{j \in N_{i}} w_{i j}\left(\delta_{j v}(t)-\delta_{i v}(t)\right)-\dot{f}_{i v}(t) .
\end{array}\right.
$$

Let $\delta_{i}(t)=\left[\delta_{i x}(t), \delta_{i v}(t)\right]^{T}$ and $\delta(t)=\left[\delta_{1}^{T}(t), \delta_{2}^{T}(t)\right.$, $\left.\ldots, \delta_{N}^{T}(t)\right]^{T}$; then, multiagent system (3) can be rewritten in the Kronecker form as follows:

$$
\begin{aligned}
\dot{\delta}(t)= & \left(L \otimes\left[\begin{array}{rr}
0 & 0 \\
-k_{1} & -k_{2}
\end{array}\right]+I_{N} \otimes\left[\begin{array}{ll}
0 & 1 \\
0 & 0
\end{array}\right]\right) \delta(t) \\
& +\left(I_{N} \otimes\left[\begin{array}{ll}
0 & 1 \\
0 & 0
\end{array}\right]\right) f(t)-\dot{f}(t) .
\end{aligned}
$$

Since the communication topology is undirected and connected, the corresponding Laplacian matrix is symmetric and has one zero eigenvalue. Let $\lambda_{1}, \lambda_{2}, \ldots, \lambda_{N}$ be the eigenvalues of the Laplacian matrix $L$ with $0=\lambda_{1}<\lambda_{2} \leq \cdots \leq \lambda_{N}$; then, there exists an orthonormal matrix $Q=\left[1_{N} / \sqrt{N}, \widetilde{Q}\right]$ such that $Q^{T} \mathrm{LQ}=\Lambda=\operatorname{diag}\left\{\lambda_{1}, \quad \lambda_{2}, \ldots, \lambda_{N}\right\}$. Let $\widetilde{\delta}(t)=\left(Q^{T} \otimes I_{2}\right) \delta(t)=\left[\widetilde{\delta}_{1}^{T}(t), \widetilde{\delta}_{2}^{T}(t), \ldots, \widetilde{\delta}_{N}^{T}(t)\right]^{T}$, and then multiagent system (4) is transformed into

$$
\begin{aligned}
& \dot{\tilde{\delta}}_{1}(t)=\left[\begin{array}{ll}
0 & 1 \\
0 & 0
\end{array}\right] \widetilde{\delta}_{1}(t)+\left(\frac{1_{N}^{T}}{\sqrt{N}} \otimes\left[\begin{array}{ll}
0 & 1 \\
0 & 0
\end{array}\right]\right) f(t)-\left(\frac{1_{N}^{T}}{\sqrt{N}} \otimes I_{2}\right) \dot{f}(t), \\
& \dot{\widetilde{\delta}}_{i}(t)=\left[\begin{array}{cc}
0 & 1 \\
-\lambda_{i} k_{1} & -\lambda_{i} k_{2}
\end{array}\right] \widetilde{\delta}_{i}(t)+\left(e_{i}^{T} Q^{T} \otimes\left[\begin{array}{ll}
0 & 1 \\
0 & 0
\end{array}\right]\right) f(t)-\left(e_{i}^{T} Q^{T} \otimes I_{2}\right) \dot{f}(t),
\end{aligned}
$$

where $\left(i \in\{2,3, \ldots, N)\right.$ and $e_{i}$ is an $N$-dimensional column with the $i$-th element being 1 and the others being 0 .

In the following theorem, a design approach of control gains $k_{1}$ and $k_{2}$ is given to achieve the ETVF.
Theorem 1. For any given $J_{u}^{*}>0$, multiagent system (1) with formation control protocol (2) achieves the ETVF if $f_{i v}(t)-$ $\dot{f}_{i x}(t)=0, \dot{f}_{i v}(t)=0(i=1,2, \ldots, N)$ and control gains $k_{1}$ and $k_{2}$ satisfy

$$
\left\{\begin{array}{l}
0<k_{1}<\min \left(-\lambda_{\max } k_{2}^{2}+2 k_{2} \kappa^{-1}, \frac{J_{u}^{*}-k_{2}^{2} \lambda_{\max } \delta_{x}^{T}(0) \delta_{x}(0)-2 k_{2} \lambda_{\max }\left|\delta_{x}^{T}(0) \delta_{v}(0)\right|-2 \delta_{v}^{T}(0) \delta_{v}(0)}{2 \lambda_{\max } \delta_{x}^{T}(0) \delta_{x}(0)}\right), \\
0<k_{2}<2 \lambda_{\max }^{-1} \kappa^{-1} .
\end{array}\right.
$$

Proof. One can set that

$$
\begin{aligned}
& \widetilde{\delta}_{c}(t)=\left(Q \otimes I_{2}\right)\left[\widetilde{\delta}_{1}^{T}(t), 0\right]^{T}=\frac{1_{N}}{\sqrt{N}} \otimes \widetilde{\delta}_{1}(t) \\
& \widetilde{\delta}_{\bar{c}}(t)=\left(Q \otimes I_{2}\right)\left[0, \widetilde{\delta}_{2}^{T}(t), \ldots, \widetilde{\delta}_{N}^{T}(t)\right]^{T}=\sum_{i=2}^{N} Q e_{i} \otimes \widetilde{\delta}_{i}(t) .
\end{aligned}
$$

Because $Q$ is nonsingular, $\widetilde{\delta}_{c}(t)$ and $\widetilde{\delta}_{\bar{c}}(t)$ are linearly independent by (8) and (9), and then we can obtain

$$
\widetilde{\delta}(t)=\widetilde{\delta}_{c}(t)+\widetilde{\delta}_{\bar{c}}(t) .
$$

According to the structure of $\widetilde{\delta}_{c}(t)$ given in $(8), \delta_{i x}(t)=$ $x_{i}(t)-f_{i x}(t)$ and $\delta_{i v}(t)=v_{i}(t)-f_{i v}(t) \quad(i=1,2, \ldots, N)$, $1 / \sqrt{N} \widetilde{\delta}_{1}(t)$ can be used as a candidate of the formation center function $c(t)$ with $c(t)=\left[c_{x}(t), c_{v}(t)\right]^{T}$, and multiagent system (1) with control protocol (2) achieves the desired formation structure if and only if $\lim _{t \rightarrow+\infty} \widetilde{\delta}_{i}(t)=0(i=2,3, \ldots, N)$.

In order to design control gains $k_{1}$ and $k_{2}$ to satisfy $\lim _{t \longrightarrow+\infty} \widetilde{\delta}_{i}(t)=0(i=2,3, \ldots, N)$, the Lyapunov function is chosen as follows: 


$$
V_{i}(t)=\widetilde{\delta}_{i}^{T}(t) \Omega_{i} \widetilde{\delta}_{i}(t)
$$

where the matrix $\Omega_{i}=\left[\begin{array}{cc}\lambda_{i}^{2} k_{2}^{2}+2 \lambda_{i} k_{1} & \lambda_{i} k_{2} \\ \lambda_{i} k_{2} & 2\end{array}\right]$. Because $\lambda_{i}>0$ and control gains $k_{1}, k_{2}>0$, it can be obtained that $\lambda_{i}^{2} k_{2}^{2}+$ $2 \lambda_{i} k_{1}>0$ and $\operatorname{det}\left(\Omega_{i}\right)=\lambda_{i}^{2} k_{2}^{2}+4 \lambda_{i} k_{1}>0$. In this case, the matrix $\Omega_{i}$ is positive definite, and the Lyapunov function $V_{i}(t)>0$ if $\widetilde{\delta}_{i}(t) \neq 0$. The time derivative of $V_{i}(t)$ along with (6) is

$$
\begin{aligned}
\dot{V}_{i}(t)= & -2 \lambda_{i}^{2} k_{1} k_{2} \widetilde{\delta}_{i x}^{2}(t)-2 \lambda_{i} k_{2} \widetilde{\delta}_{i v}^{2}(t)+2 \widetilde{\delta}_{i}^{T}(t) \Omega_{i} \\
& \times\left(\left(e_{i}^{T} Q^{T} \otimes\left[\begin{array}{ll}
0 & 1 \\
0 & 0
\end{array}\right]\right) f(t)-\left(e_{i}^{T} Q^{T} \otimes I_{2}\right) \dot{f}(t)\right),
\end{aligned}
$$

where one can find that $-2 \lambda_{i}^{2} k_{1} k_{2}<0$ and $-2 \lambda_{i} k_{2}<0$. Since

$$
\left(e_{i}^{T} Q^{T} \otimes\left[\begin{array}{ll}
0 & 1 \\
0 & 0
\end{array}\right]\right) f(t)=\left(\left(e_{i}^{T} Q^{T} \otimes I_{2}\right)\left(I_{N} \otimes\left[\begin{array}{ll}
0 & 1 \\
0 & 0
\end{array}\right]\right)\right) f(t)
$$

we have

$$
\begin{aligned}
& \left(e_{i}^{T} Q^{T} \otimes\left[\begin{array}{ll}
0 & 1 \\
0 & 0
\end{array}\right]\right) f(t)-\left(e_{i}^{T} Q^{T} \otimes I_{2}\right) \dot{f}(t) \\
& \quad=\left(e_{i}^{T} Q^{T} \otimes I_{2}\right)\left(\left(I_{N} \otimes\left[\begin{array}{ll}
0 & 1 \\
0 & 0
\end{array}\right]\right) f(t)-\dot{f}(t)\right) .
\end{aligned}
$$

It can be found that $Q^{T} \otimes I_{2}$ is nonsingular, and if $f_{i v}(t)-\dot{f}_{i x}(t)=0, \dot{f}_{i v}(t)=0(i=1,2, \ldots, N)$, then one can obtain that

$$
\left(e_{i}^{T} Q^{T} \otimes\left[\begin{array}{ll}
0 & 1 \\
0 & 0
\end{array}\right]\right) f(t)-\left(e_{i}^{T} Q^{T} \otimes I_{2}\right) \dot{f}(t)=0 .
$$

According to (12) and (15), it is verified that $\dot{V}_{i}(t)<0$ if $\widetilde{\delta}_{i}(t) \neq 0$; then, one can obtain that $\lim _{t \longrightarrow+\infty} \widetilde{\delta}_{i}(t)=0(i=2,3, \ldots, N)$. Hence, multiagent system (1) with formation control protocol (2) can achieve the desired time-varying formation.

The next step is to focus on the problem of the energylimited formation. It can be seen that

$$
\sum_{i=1}^{N} u_{i}^{2}(t)=\delta^{T}(t)\left(L^{2} \otimes\left[\begin{array}{cc}
k_{1}^{2} & k_{1} k_{2} \\
k_{1} k_{2} & k_{2}^{2}
\end{array}\right]\right) \delta(t) .
$$

Since $\delta(t)=\left(Q \otimes I_{2}\right) \widetilde{\delta}(t)$ and $\lambda_{1}=0$, (16) can be converted into

$$
\widetilde{\delta}^{T}(t)\left(\Lambda^{2} \otimes\left[\begin{array}{cc}
k_{1}^{2} & k_{1} k_{2} \\
k_{1} k_{2} & k_{2}^{2}
\end{array}\right]\right) \widetilde{\delta}(t)=\sum_{i=2}^{N} \lambda_{i}^{2} \widetilde{\delta}_{i}^{T}(t)\left[\begin{array}{cc}
k_{1}^{2} & k_{1} k_{2} \\
k_{1} k_{2} & k_{2}^{2}
\end{array}\right] \delta_{i}(t) .
$$

From (16) and (17), it can be seen that

$$
\begin{aligned}
J_{\Delta} & =\int_{0}^{\Delta} \sum_{i=1}^{N} \kappa u_{i}^{2}(t) \mathrm{d} t \\
& =\int_{0}^{\Delta} \kappa\left(\sum_{i=2}^{N} \lambda_{i}^{2}\left(k_{1} \widetilde{\delta}_{i x}(t)+k_{2} \widetilde{\delta}_{i v}(t)\right)^{2}\right) \mathrm{d} t,
\end{aligned}
$$

where $\Delta \geq 0$. Because $\int_{0}^{\Delta} \dot{V}_{i}(t) d t=V_{i}(\Delta)-V_{i}(0)$, one can get

$$
\begin{aligned}
J_{\Delta} & =\int_{0}^{\Delta} \kappa\left(\sum_{i=2}^{N} \lambda_{i}^{2}\left(k_{1} \widetilde{\delta}_{i x}(t)+k_{2} \widetilde{\delta}_{i v}(t)\right)^{2}\right) \mathrm{d} t+\sum_{i=2}^{N}\left(\int_{0}^{\Delta} \dot{V}_{i}(t) \mathrm{d} t-V_{i}(\Delta)+V_{i}(0)\right) \\
& =\sum_{i=2}^{N}\left(\int_{0}^{\Delta}\left(\left(\kappa \lambda_{i}^{2} k_{1}^{2}-2 \lambda_{i}^{2} k_{1} k_{2}\right) \tilde{\delta}_{i x}^{2}(t)+2 \kappa \lambda_{i}^{2} k_{1} k_{2} \widetilde{\delta}_{i x}(t) \widetilde{\delta}_{i v}(t)+\left(\kappa \lambda_{i}^{2} k_{2}^{2}-2 \lambda_{i} k_{2}\right) \widetilde{\delta}_{i v}^{2}(t)\right) \mathrm{d} t-V_{i}(\Delta)\right)+\sum_{i=2}^{N} V_{i}(0) .
\end{aligned}
$$

Let

$$
\Xi_{i}=\left[\begin{array}{cc}
\kappa \lambda_{i}^{2} k_{1}^{2}-2 \lambda_{i}^{2} k_{1} k_{2} & \kappa \lambda_{i}^{2} k_{1} k_{2} \\
\kappa \lambda_{i}^{2} k_{1} k_{2} & \kappa \lambda_{i}^{2} k_{2}^{2}-2 \lambda_{i} k_{2}
\end{array}\right] .
$$

If $\Xi_{i}<0$, then $\quad \kappa \lambda_{i}^{2} k_{1}^{2}-2 \lambda_{i}^{2} k_{1} k_{2}<0 \quad$ and $\left(\kappa \lambda_{i}^{2} k_{1}^{2}-2 \lambda_{i}^{2} k_{1} k_{2}\right)\left(\kappa \lambda_{i}^{2} k_{2}^{2}-2 \lambda_{i} k_{2}\right)-\kappa^{2} \lambda_{i}^{4} k_{1}^{2} k_{2}^{2}>0$. Hence, it can be concluded that the value range of control gains $k_{1}$ and $k_{2}: 0<k_{1}<-\lambda_{\max } k_{2}^{2}+2 k_{2} \kappa^{-1}, 0<k_{2}<2 \lambda_{\max }^{-1} \kappa^{-1}$. In this case, the matrix $\Xi_{i}$ is negative definite, and from (12) to (19), one can find that

$$
J_{u} \leq \sum_{i=2}^{N} V_{i}(0)
$$

Because $\tilde{\delta}(t)=\left(Q^{T} \otimes I_{2}\right) \delta(t)$, one can obtain that $\widetilde{\delta}_{x}(t)=Q^{T} \delta_{x}(t) \quad$ and $\quad \tilde{\delta}_{v}(t)=Q^{T} \delta_{v}(t)$, where $\widetilde{\delta}_{x}(t)=\left[\widetilde{\delta}_{1 x}(t), \widetilde{\delta}_{2 x}(t), \ldots, \widetilde{\delta}_{N x}(t)\right]^{T}$, $\widetilde{\delta}_{v}(t)=\left[\widetilde{\delta}_{1 v}(t), \widetilde{\delta}_{2 v}(t), \ldots, \widetilde{\delta}_{N v}(t)\right]^{T}, \delta_{x}(t)=\left[\delta_{1 x}(t), \delta_{2 x}(t)\right.$, $\left.\ldots, \delta_{N x}(t)\right]^{T}$, and $\delta_{v}(t)=\left[\delta_{1 v}(t), \delta_{2 v}(t), \ldots, \delta_{N v}(t)\right]^{T}$. Moreover, (21) can be rewritten as

$$
\begin{aligned}
\sum_{i=2}^{N} V_{i}(0)= & \sum_{i=2}^{N}\left(\lambda_{i}^{2} k_{2}^{2}+2 \lambda_{i} k_{1}\right) \widetilde{\delta}_{i x}^{2}(0)+2 \lambda_{i} k_{2} \widetilde{\delta}_{i x}(0) \widetilde{\delta}_{i v}(t)+2 \widetilde{\delta}_{i v}^{2}(0) \\
= & k_{2}^{2} \widetilde{\delta}_{x}^{T}(0) Q^{T} L^{2} Q \widetilde{\delta}_{x}(0)+2 k_{1} \widetilde{\delta}_{x}^{T}(0) Q^{T} L Q \widetilde{\delta}_{x}(0) \\
& +2 k_{2} \widetilde{\delta}_{x}^{T}(0) Q^{T} L Q \widetilde{\delta}_{v}(0)+\sum_{i=2}^{N} 2 \widetilde{\delta}_{i v}^{2}(0) \\
\leq & k_{2}^{2} \lambda_{\max }^{2} \delta_{x}^{T}(0) \delta_{x}(0)+2 k_{1} \lambda_{\max } \delta_{x}^{T}(0) \delta_{x}(0)+2 k_{2} \lambda_{\max } \\
& \cdot\left|\delta_{x}^{T}(0) \delta_{v}(0)\right| \\
& +2 \delta_{v}^{T}(0) \delta_{v}(0) .
\end{aligned}
$$


From (21) to (22), one can obtain that

$$
\begin{aligned}
J_{u} \leq & k_{2}^{2} \lambda_{\max }^{2} \delta_{x}^{T}(0) \delta_{x}(0)+2 k_{1} \lambda_{\max } \delta_{x}^{T}(0) \delta_{x}(0)+2 k_{2} \lambda_{\max } \\
\cdot & \left|\delta_{x}^{T}(0) \delta_{v}(0)\right| 2 \delta_{v}^{T}(0) \delta_{v}(0) .
\end{aligned}
$$

In this case, there exists

$$
k_{1} \leq \frac{J_{u}^{*}-k_{2}^{2} \lambda_{\max }^{2} \delta_{x}^{T}(0) \delta_{x}(0)-2 k_{2} \lambda_{\max }\left|\delta_{x}^{T}(0) \delta_{v}(0)\right|-2 \delta_{v}^{T}(0) \delta_{v}(0)}{2 \lambda_{\max } \delta_{x}^{T}(0) \delta_{x}(0)},
$$

such that $J_{u} \leq J_{u}^{*}$. Hence, the conclusion of Theorem 1 is drawn. It is worth noting that the conclusion is related to the maximum eigenvalue of the topology. Based on the theoretical analysis, the stability of the system is closely related to the Laplacian matrix, and then it can be converted into the conclusion about the eigenvalues of the Laplacian matrix.

Remark 3. Theoretically, any desired geometric structure formation can be realized by selecting the corresponding formation function. However, from the perspective of mechanism modeling, the feasibility condition of the formation is restricted by the structure of dynamic systems in practice. Accordingly, it is significant to choose a proper time-varying formation function that meets the formation feasibility constraint condition $\left[\begin{array}{ll}0 & 1 \\ 0 & 0\end{array}\right] f_{i}(t)-\dot{f}_{i}(t)=0$ $(i=1,2, \ldots, N)$, that is, $f_{i v}(t)-\dot{f}_{i x}(t)=0, \dot{f}_{i v}(t)=0$ $(i=1,2, \ldots, N)$, which is echoed by the dynamics of each agent (1). In this case, the derivative of the position component of the formation function is equal to its velocity component, and the center of the formation moves in a uniform straight line. Note that if $\dot{f}_{i}(t) \equiv 0(i=1,2, \ldots, N)$, then the formation feasibility constraint condition is $\left[\begin{array}{ll}0 & 1 \\ 0 & 0\end{array}\right] f_{i}(t)=0(i=1,2, \ldots, N)$, where the formation is time-invariant. In this case, the time-invariant formation only forms a certain static geometric formation structure, but the time-varying one not only forms a certain structure but also moves in a desired geometric formation according to the requirement of the realistic environments. On the whole, it can be illustrated that the feasible condition of the time-varying formation has wide scope, while for the timeinvariant formation, it is just a special case.

Remark 4. In most works, the control gain is obtained by the FEASP solver of Matlab's LMI Toolbox, which is a numerical solution algorithm. However, it is difficult and unable for the FEASP solver to find feasible solutions in some situations, which makes solutions severely limited. Therefore, it is necessary to find the analytical solutions of $k_{1}$ and $k_{2}$ that do not need to verify their feasibility. In this case, the nonlinear relationship of the control gains $k_{1}$ and $k_{2}$ is separated. It can be seen that $k_{2}$ is determined independently, and $k_{1}$ relies on $k_{2}$, and the value ranges of control gains $k_{1}$ and $k_{2}$ can be simultaneously determined by using the function monotony. Therefore, one can choose any value in the range of control gains $k_{1}$ and $k_{2}$, instead of trying to find a certain existing value that must satisfy the feasibility.

From Theorem 1, one can find that the candidate of the formation center function is given as $c(t)=1 / \sqrt{N} \widetilde{\delta}_{1}(t)$, and a method to determine the formation center function is presented in the following theorem.

Theorem 2. If multiagent system (1) under formation control protocol (2) achieves the ETVF determined by $f_{i}(t)(i=1,2, \ldots, N)$, then the formation center function $c(t)$ satisfies that

$\lim _{t \rightarrow \infty}\left(c(t)-\frac{1}{N}\left(e^{\left.\left[\begin{array}{ll}0 & 1 \\ 0 & 0\end{array}\right]^{t} \sum_{i=1}^{N}\left[x_{i}(0), v_{i}(0)\right]^{T}+\sum_{i=1}^{N} f_{i}(t)\right)}=0\right.\right.$.

Proof. Since $Q=\left[1_{N} / \sqrt{N}, \widetilde{Q}\right]$ and $\tilde{\delta}(t)=\left(Q^{T} \otimes I_{2}\right) \delta(t)$, one can find that

$$
\widetilde{\delta}_{1}(0)=\left(\frac{1_{N}^{T}}{\sqrt{N}} \otimes I_{2}\right) \delta(0)=\frac{1}{\sqrt{N}} \sum_{i=1}^{N} \delta_{i}(0)
$$

According to $\delta_{i}(t)=\left[\delta_{i x}(t), \delta_{i v}(t)\right]^{T}$ with $\delta_{i x}(t)=$ $x_{i}(t)-f_{i x}(t)$ and $\delta_{i v}(t)=v_{i}(t)-f_{i v}(t)(i=1,2, \ldots, N)$, (26) is converted into

$$
\widetilde{\delta}_{1}(0)=\frac{1}{\sqrt{N}}\left(\sum_{i=1}^{N}\left[x_{i}(0), v_{i}(0)\right]^{T}-\sum_{i=1}^{N} f_{i}(0)\right) .
$$

In the following, the dynamic response of subsystem (5) is obtained by $\widetilde{\delta}_{1}(0), f(t)$, and $\dot{f}(t)$, respectively. 


$$
\begin{gathered}
c_{0}(t)=\frac{1}{\sqrt{N}} e^{\left[\begin{array}{ll}
0 & 1 \\
0 & 0
\end{array}\right] t}\left(\sum_{i=1}^{N}\left[x_{i}(0), v_{i}(0)\right]^{T}-\sum_{i=1}^{N} f_{i}(0)\right), \\
c_{f}(t)=\int_{0}^{t} e^{\left[\begin{array}{ll}
0 & 1 \\
0 & 0
\end{array}\right](t-\tau)}\left(\frac{1_{N}^{T}}{\sqrt{N}} \otimes\left[\begin{array}{ll}
0 & 1 \\
0 & 0
\end{array}\right]\right) f(\tau) \mathrm{d} \tau, \\
c_{\dot{f}}(t)=-\int_{0}^{t} e^{\left[\begin{array}{ll}
0 & 1 \\
0 & 0
\end{array}\right](t-\tau)}\left(\frac{1_{N}^{T}}{\sqrt{N}} \otimes I_{2}\right) \dot{f}(\tau) \mathrm{d} \tau .
\end{gathered}
$$

Because

$$
\begin{aligned}
\left(1_{N}^{T} \otimes I_{2}\right) f(t)= & \sum_{i=1}^{N} f_{i}(t), \\
& {\left[\begin{array}{ll}
0 & 1 \\
0 & 0
\end{array}\right] e^{\left[\begin{array}{ll}
0 & 1 \\
0 & 0
\end{array}\right](t-\tau)}\left(1_{N}^{T} \otimes I_{2}\right) f(\tau) } \\
& =e^{\left[\begin{array}{ll}
0 & 1 \\
0 & 0
\end{array}\right](t-\tau)}\left(1_{N}^{T} \otimes\left[\begin{array}{ll}
0 & 1 \\
0 & 0
\end{array}\right]\right) f(\tau),
\end{aligned}
$$

by (28), (29), and (30), one can obtain that the dynamic response of subsystem (5) can be written as follows:

$$
\widetilde{\delta}_{1}(t)=\frac{1}{\sqrt{N}} e^{\left[\begin{array}{ll}
0 & 1 \\
0 & 0
\end{array}\right] t} \sum_{i=1}^{N}\left[x_{i}(0), v_{i}(0)\right]^{T}-\frac{1}{\sqrt{N}} \sum_{i=1}^{N} f_{i}(t) \text {. }
$$

Furthermore, the formation center function is

$$
c(t)=\frac{1}{\sqrt{N}} \widetilde{\delta}_{1}(t)=\frac{1}{N} e^{\left[\begin{array}{ll}
0 & 1 \\
0 & 0
\end{array}\right]} \sum_{i=1}^{N}\left[x_{i}(0), v_{i}(0)\right]^{T}-\frac{1}{N} \sum_{i=1}^{N} f_{i}(t) \text {. }
$$

From (26) to (33), the conclusion of Theorem 2 is drawn.

Remark 5. The most critical problem is to develop the formula of formation center function $c(t)$ in Theorem 2 . The formula is composed of the initial state and the formation function, in which the former is connected with the average value of initial states $x_{i}(0)$ and $v_{i}(0)$ and the dynamics of each agent and the latter is associated with the average value of the formation function. Under this circumstance, the formation center function $c(t)$ is located at the geometric center of the whole formation structure, which depicts the whole macroscopic motion, and the formation function $f_{i}(t)(i=1,2, \ldots, N)$ denotes expected formation state, which describes relative microscopic motion among agents. Besides, formation center function $c(t)$ has nothing to do with the derivative of the formation function, and energy limitation does not affect the formation center function.

\section{ETVF Control for the Leader-Following Case}

The energy-limited multiagent system consisting of a leader and $N-1$ followers is focused on in this section. The dynamics of the leader is shown as

$$
\left\{\begin{array}{l}
\dot{x}_{1}(t)=v_{1}(t) \\
\dot{v}_{1}(t)=0
\end{array}\right.
$$

where $x_{1}(t)$ and $v_{1}(t)$ are the position and velocity terms of the leader, and the leader does not obtain any state information from all followers. The dynamics of the followers is given by

$$
\left\{\begin{array}{l}
\dot{x}_{i}(t)=v_{i}(t) \\
\dot{v}_{i}(t)=u_{i}(t)
\end{array}\right.
$$

where $i=2,3, \ldots, N$. For each follower, there is at least one path to get the state information from the leader, and the leader as a reference object for all followers is uncontrolled; it is only a one-way transmission. Let $f_{1}(t) \equiv 0$; then, the formation control protocol for the energy-limited formation is given as follows:

$$
\left\{\begin{array}{l}
u_{i}(t)=w_{i 1} k_{1}\left(x_{1}(t)-x_{i}(t)+f_{i x}(t)\right)+w_{i 1} k_{2}\left(v_{1}(t)-v_{i}(t)+f_{i v}(t)\right) \\
+\sum_{j \in N_{i}, j \neq 1} w_{i j}\left(k_{1}\left(x_{j}(t)-f_{j x}(t)-x_{i}(t)+f_{i x}(t)\right)+k_{2}\left(v_{j}(t)-f_{j v}(t)-v_{i}(t)+f_{i v}(t)\right)\right), \\
J_{u}=\sum_{i=2}^{N} \int_{0}^{+\infty} \kappa u_{i}^{2}(t) \mathrm{d} t .
\end{array}\right.
$$

The states of followers are required to maintain a timevarying formation determined by the formation function $f_{i}(t)=\left[f_{i x}(t), f_{i v}(t)\right]^{T}$ and to track the states of the leader. Suppose that the formation center function $\bar{c}(t)=\left[x_{1}(t), v_{1}(t)\right]^{T}$ for the leader-following case, where $x_{1}(t)$ and $v_{1}(t)$ correspond to the position and velocity terms of the leader, respectively.

Let $\psi_{i x}(t)=x_{i}(t)-f_{i x}(t)$ and $\psi_{i v}(t)=v_{i}(t)-f_{i v}$ (t) $(i=2,3, \ldots, N)$; then, the dynamics of multiagent system (35) under formation control protocol (36) can be shown as 


$$
\left\{\begin{array}{l}
\dot{\psi}_{i x}(t)=\psi_{i v}(t)+f_{i v}(t)-\dot{f}_{i x}(t), \\
\dot{\psi}_{i v}(t)=k_{1} w_{i 1} x_{1}(t)+k_{2} w_{i 1} v_{1}(t)+k_{1} \sum_{j \in N_{i}, j \neq 1} w_{\sigma(t), i j}\left(\psi_{j x}(t)-\psi_{i x}(t)\right) \\
+k_{2} \sum_{j \in N_{i}, j \neq 1} w_{\sigma(t), i j}\left(\psi_{j v}(t)-\psi_{i v}(t)\right)-\dot{f}_{i v}(t) .
\end{array}\right.
$$

Let $\quad \psi_{i}(t)=\left[\psi_{i x}(t), \psi_{i v}(t)\right]^{T}(i=2,3, \ldots, N) \quad$ and $\psi(t)=\left[\bar{c}^{T}(t), \psi_{2}^{T}(t), \ldots, \psi_{N}^{T}(t)\right]^{T}$; then, multiagent system (35) can be rewritten in the following Kronecker form:

$$
\begin{aligned}
\dot{\psi}(t)= & \left(L \otimes\left[\begin{array}{rr}
0 & 0 \\
-k_{1} & -k_{2}
\end{array}\right]+I_{N} \otimes\left[\begin{array}{ll}
0 & 1 \\
0 & 0
\end{array}\right]\right) \psi(t) \\
& +\left(I_{N} \otimes\left[\begin{array}{ll}
0 & 1 \\
0 & 0
\end{array}\right]\right) f(t)-\dot{f}(t),
\end{aligned}
$$

where the Laplacian matrix $L=\left[\begin{array}{cc}0 & 0 \\ -l_{\mathrm{fl}} & L_{\mathrm{ff}}+\Lambda_{\mathrm{fl}}\end{array}\right]$ with $l_{\mathrm{fl}}=\left[w_{21}, w_{31}, \ldots, w_{N 1}\right]^{T}, \quad \Lambda_{\mathrm{fl}}=\operatorname{diag}\left\{w_{21}, w_{31}, \ldots, w_{N 1}\right\}$, and $L_{\mathrm{ff}}$ represents the Laplacian matrix among the followers. that

Let the matrix $\widehat{T}=\left[\begin{array}{cc}1 & 0 \\ 1_{N-1} & I_{N-1}\end{array}\right]$; then, one can obtain

$$
\widehat{T}^{-1} L \widehat{T}=\left[\begin{array}{ll}
0 & \\
& L_{\mathrm{ff}}+\Lambda_{\mathrm{fl}}
\end{array}\right] .
$$

Build the state differences of the leader and each follower $\bar{\psi}_{i}\left(t_{1}\right)=\psi_{i}(t)-\bar{c}(t)(i=2,3, \ldots, N)$; one can find that $\left(T \otimes I_{2}\right) \psi(t)=\left[\bar{c}^{T}(t), \bar{\psi}^{T}(t)\right]^{T}$ with $\bar{\psi}(t)=\left[\bar{\psi}_{2}^{T}(t), \bar{\psi}_{3}^{T}(t)\right.$, $\left.\ldots, \bar{\psi}_{N}^{T}(t)\right]^{T}$. Since the local communication topology among followers is undirected, the Laplacian matrix $L_{\mathrm{ff}}+\Lambda_{\mathrm{fl}}$ is symmetric. Without loss of generality, suppose that $\widehat{\lambda}_{2}, \widehat{\lambda}_{3}, \ldots, \widehat{\lambda}_{N}>0(i=2,3, \ldots, N)$ are eigenvalues of Laplacian matrix $L_{\mathrm{ff}}+\Lambda_{\mathrm{fl}}$. Under the circumstances, there exists an orthonormal matrix $\widetilde{Q}$ such that $\widetilde{Q}^{T}\left(L_{\mathrm{ff}}+\Lambda_{\mathrm{fl}}\right) \widetilde{Q}=\widetilde{\Lambda}$, where $\widetilde{\Lambda}=\operatorname{diag}\left\{\widehat{\lambda}_{2}, \widehat{\lambda}_{3}, \ldots, \hat{\lambda}_{N}\right\}$ Let $\left(\widetilde{Q}^{T} \otimes I_{2}\right)$
$\bar{\psi}(t)=\widetilde{\psi}(t)=\left[\widetilde{\psi}_{2}^{T}(t), \ldots, \widetilde{\psi}_{N}^{T}(t)\right]^{T}$; then, (38) can be converted into

$$
\begin{aligned}
\dot{\bar{c}}(t)= & {\left[\begin{array}{ll}
0 & 1 \\
0 & 0
\end{array}\right] \bar{c}(t), } \\
\dot{\widetilde{\psi}}(t)= & {\left[\begin{array}{cc}
0 & 1 \\
-\widehat{\lambda}_{i} k_{1} & -\widehat{\lambda}_{i} k_{2}
\end{array}\right] \widetilde{\psi}_{i}(t) } \\
& +\left(e_{i-1}^{T} \widetilde{Q}^{T}\left[0, I_{N-1}\right] \widehat{T}^{-1} \otimes\left[\begin{array}{ll}
0 & 1 \\
0 & 0
\end{array}\right]\right) f(t) \\
& -\left(e_{i-1}^{T} \widetilde{Q}^{T}\left[0, I_{N-1}\right] \widehat{T}^{-1} \otimes I_{2}\right) \dot{f}(t),
\end{aligned}
$$

where $i=2,3, \ldots, N, \bar{c}(t)$ is the formation center function, and multiagent (1) under formation control protocol (2) achieves the desired formation.

Next, it can be found that

$$
\sum_{i=2}^{N} u_{i}^{2}(t)=\bar{\psi}^{T}(t)\left(\left(L_{f f}+\Lambda_{f l}\right)^{2} \otimes\left[\begin{array}{cc}
k_{1}^{2} & k_{1} k_{2} \\
k_{1} k_{2} & k_{2}^{2}
\end{array}\right]\right) \bar{\psi}(t) .
$$

Since $\left(\widetilde{Q}^{T} \otimes I_{2}\right) \bar{\psi}(t)=\widetilde{\psi}(t),(41)$ can be transformed into $\widetilde{\psi}^{T}(t)\left(\widetilde{\Lambda}^{2} \otimes\left[\begin{array}{cc}k_{1}^{2} & k_{1} k_{2} \\ k_{1} k_{2} & k_{2}^{2}\end{array}\right]\right) \widetilde{\psi}(t)=\sum_{i=2}^{N} \hat{\lambda}_{i}^{2}\left(k_{1} \widetilde{\psi}_{i x}(t)+k_{2} \widetilde{\psi}_{i v}(t)\right)^{2}$.

Let $\quad \bar{\psi}_{x}(t)=\left[\bar{\psi}_{2 x}(t), \bar{\psi}_{3 x}(t), \ldots, \bar{\psi}_{N x}(t)\right]^{T} \quad$ and $\bar{\psi}_{v}(t)=\left[\bar{\psi}_{2 v}(t), \bar{\psi}_{3 v}(t), \ldots, \bar{\psi}_{N v}(t)\right]^{T}$; one can obtain that

$$
\begin{aligned}
\sum_{i=2}^{N} u_{i}^{2}(t)= & k_{2}^{2} \widetilde{\psi}_{x}^{T}(t) \widetilde{Q}^{T}\left(L_{f f}+\Lambda_{f l}\right)^{2} \widetilde{Q}_{\bar{\psi}}(t)+2 k_{1} \widetilde{\psi}_{x}^{T}(t) \widetilde{Q}^{T}\left(L_{f f}+\Lambda_{f l}\right) \widetilde{Q} \widetilde{\psi}_{x}(t) \\
& +2 k_{2} \widetilde{\psi}_{x}^{T}(t) \widetilde{Q}^{T}\left(L_{f f}+\Lambda_{f l}\right) \widetilde{Q}_{\psi}(t)+2 \widetilde{\psi}_{v}^{T}(t) \widetilde{Q}^{T} \widetilde{Q}_{\psi} \widetilde{\psi}_{v}(t) \\
\leq & k_{2}^{2} \widehat{\lambda}_{\max }^{2} \bar{\psi}_{x}^{T}(0) \bar{\psi}_{x}(0)+2 k_{1} \widehat{\lambda}_{\max } \bar{\psi}_{x}^{T}(0) \bar{\psi}_{x}(0)+2 k_{2} \widehat{\lambda}_{\max }\left|\bar{\psi}_{x}^{T}(0) \bar{\psi}_{v}(0)\right| \\
& +2 \widehat{\lambda}_{\max } \bar{\psi}_{v}^{T}(0) \bar{\psi}_{v}(0) .
\end{aligned}
$$


The proof procedure from (41) to (43) is similar to the Theorem 1. Hence, the conclusions about energy-limited formation can be drawn in the following.

Theorem 3. For any given $J_{u}^{*}>0$, multiagent system (1) with formation control protocol (2) achieves the ETVF if $f_{\text {iv }}(t)-$ $\dot{f}_{i x}(t)=0, \dot{f}_{i v}(t)=0(i=2,3, \ldots, N)$ and there exist control gains $k_{1}$ and $k_{2}$ satisfying

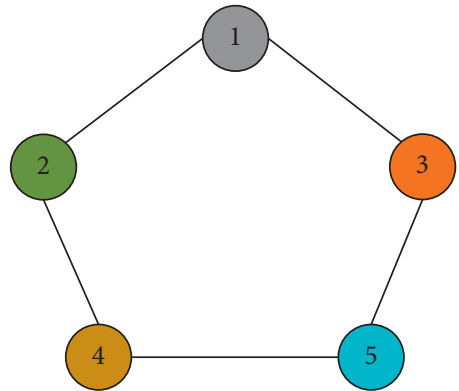

Figure 1: Communication topology $G$ for the leaderless case.

$$
\left\{\begin{array}{l}
0<k_{1}<\min \left(-\hat{\lambda}_{\max } k_{2}^{2}+2 k_{2} \kappa^{-1}, \frac{J_{u}^{*}-k_{2}^{2} \hat{\lambda}_{\max }^{2} \bar{\psi}_{x}^{T}(0) \bar{\psi}_{x}(0)-2 k_{2} \hat{\lambda}_{\max }\left|\bar{\psi}_{x}^{T}(0) \bar{\psi}_{v}(0)\right|-2 \bar{\psi}_{v}^{T}(0) \bar{\psi}_{v}(0)}{2 \widehat{\lambda}_{\max } \bar{\psi}_{x}^{T}(0) \bar{\psi}_{x}(0)}\right), \\
0<k_{2}<2 \hat{\lambda}_{\max }^{-1} \kappa^{-1} .
\end{array}\right.
$$

Remark 6. Different from the leaderless case in Theorem 1, the leader-following one has two characteristics. Firstly, the leader-following communication topology is asymmetric, which means the leader does not get any information from followers, while at least one follower can get it from the leader. Besides, the local communication topology among the followers is undirected. In this case, a two-step transformation method is proposed, wherein the most critical step is to construct state differences between followers and the leader using a special transformation, so that leaderless and leader-following cases can be unified into an identical framework and the derivation process of leader-following cases is simplified. From a macro perspective, the whole movement is determined by all agents for leaderless multiagent systems, but it only relies on the leader for leaderfollowing ones. Secondly, the range of control gains $k_{1}, k_{2}$ for leaderless case is about the initial states of each agent, but it is related to the state differences of the initial values between the leader and each follower for leader-following case, which is the state difference method proposed to convert the asymmetric matrix into a symmetric one.

\section{Numerical Simulation}

In this section, two numerical examples of leaderless and leader-following MAS are used to verify correctness and effectiveness of the theoretical results, respectively.

Example 1. (the leaderless case) Consider the leaderless MAS with five agents moving on a 2-dimensional plane (XY plane). The dynamics of each agent is expressed by (1) with $x_{i}(t)=\left[x_{i X}(t), x_{i Y}(t)\right]^{T}, \quad v_{i}(t)=\left[v_{i X}(t), v_{i Y}(t)\right]^{T}, \quad$ and $u_{i}(t)=\left[u_{i X}(t), u_{i Y}(t)\right]^{T}(i \in\{1,2, \ldots, 5\}), \quad$ in which $x_{i X}(t), v_{i X}(t), u_{i X}(t)$ and $x_{i Y}(t), v_{i Y}(t), u_{i Y}(t)$ denoting position, velocity, and control input are along the $X$-axis and $Y$ axis in the $X Y$ plane, respectively. The communication among agents is subject to the undirected topology $G$ shown in Figure 1, where the weight of the adjacent matrix is $0-1$.
The initial states $\left[x_{i}(0), v_{i}(0)\right]^{T}=\left[x_{i X}(0), x_{i Y}(0), v_{i X}(0)\right.$, $\left.v_{i Y}(0)\right]^{T}(i \in\{1,2, \ldots, 5\})$ are given as follows:

$$
\begin{aligned}
& {\left[x_{1}(0), v_{1}(0)\right]^{T}=[1.1,1.3,0.4,-1.2]^{T},} \\
& {\left[x_{2}(0), v_{2}(0)\right]^{T}=[0.2,-0.8,-1.3,0.5]^{T},} \\
& {\left[x_{3}(0), v_{3}(0)\right]^{T}=[-0.3,1.1,0.5,0.6]^{T},} \\
& {\left[x_{4}(0), v_{4}(0)\right]^{T}=[-1.3,-0.7,-1.2,1.1]^{T},} \\
& {\left[x_{5}(0), v_{5}(0)\right]^{T}=[0.9,0.8,1.1,0.2]^{T} .}
\end{aligned}
$$

The formation function for leaderless MAS is given as

$$
\begin{aligned}
f_{i}(t)= & {\left[t \sin \left(\frac{2(i-1) \pi}{5}\right), t \cos \left(\frac{2(i-1) \pi}{5}\right),\right.} \\
& \left.\sin \left(\frac{2(i-1) \pi}{5}\right), \cos \left(\frac{2(i-1) \pi}{5}\right)\right]^{T}, \quad i \in\{1,2, \ldots, 5\} .
\end{aligned}
$$

Let $\kappa=0.4$ and the total energy $J_{u}^{*}=120$; then, one can find by Theorem 1 that $0<k_{1}<0.3455$ and $0<k_{2}<0.6910$. Here, one chooses $k_{1}=0.2$ and $k_{2}=0.5$.

In Figures 2 and 3 , state trajectories of $\delta_{i x}(t)$ and $\delta_{i v}(t)(i=$ $1,2, \ldots, 5)$ for leaderless MAS along the $X$-axis and $Y$-axis are depicted, respectively, where the curve marked by blue circles refers to the trajectories of the formation center function. Figure 4 depicts snapshots of the state of five agents and a formation center at different times, where five agents are marked with a red square, a green diamond, a magenta circle, a sky blue triangle, and a black pentagon, and the blue hexagon stands for the formation center. From Figures 4(a) $-4(d)$, one can see that the pentagon formation is formed, and the pentagon formation structure is changing over time at the same multiple. Figure 5 shows the state trajectories of each agent in the whole time, and the trajectory of the formation center is given in Figure 6. Figure 7 shows that the practical energy consumption $J_{u}$ converges to a finite value with $J_{u}<J_{u}^{*}$. It is clear that the desired pentagonal time-varying formation under 

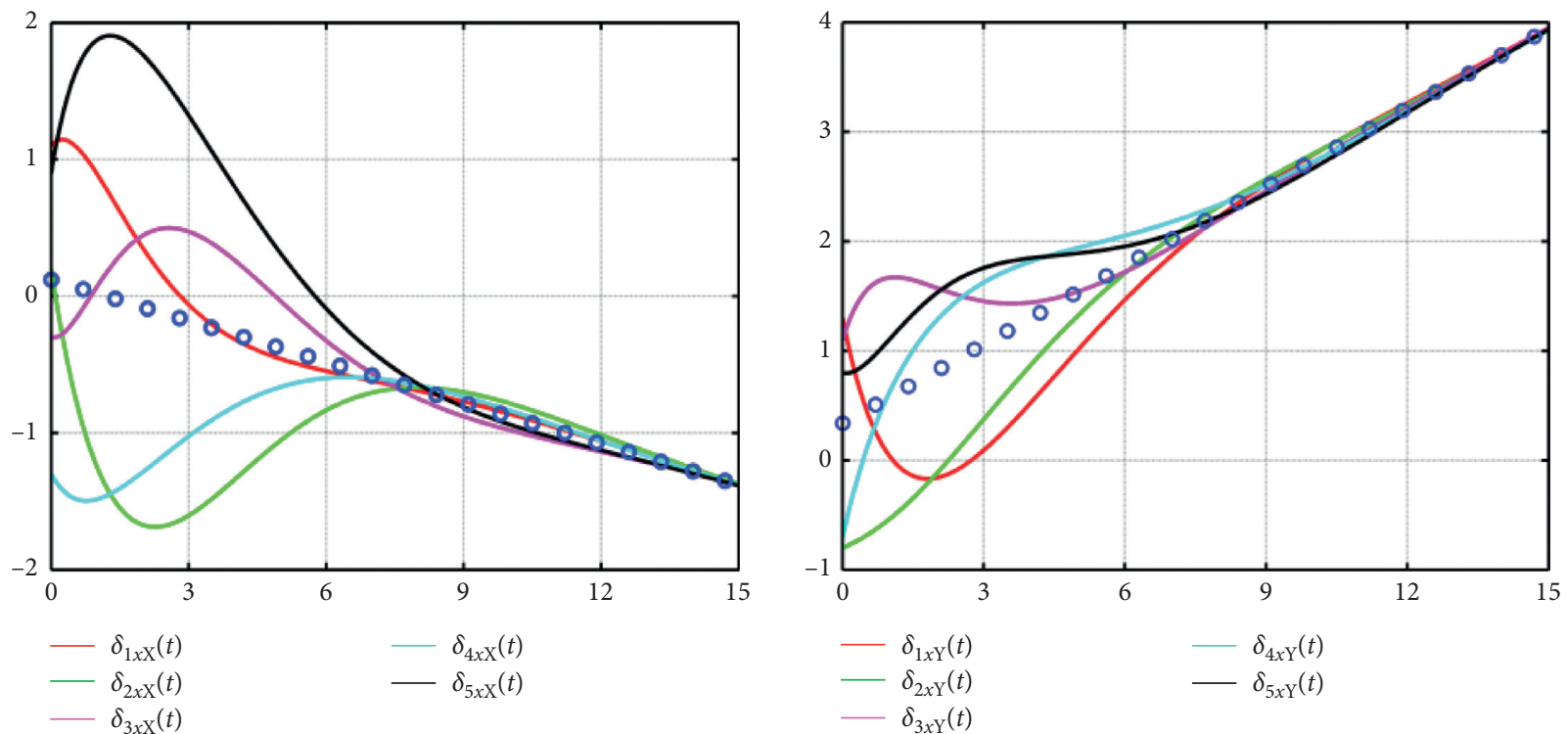

FIgURE 2: Trajectories of $\delta_{i x}(t)(i=1,2, \ldots, 5)$ in $X$ and $Y$ directions.
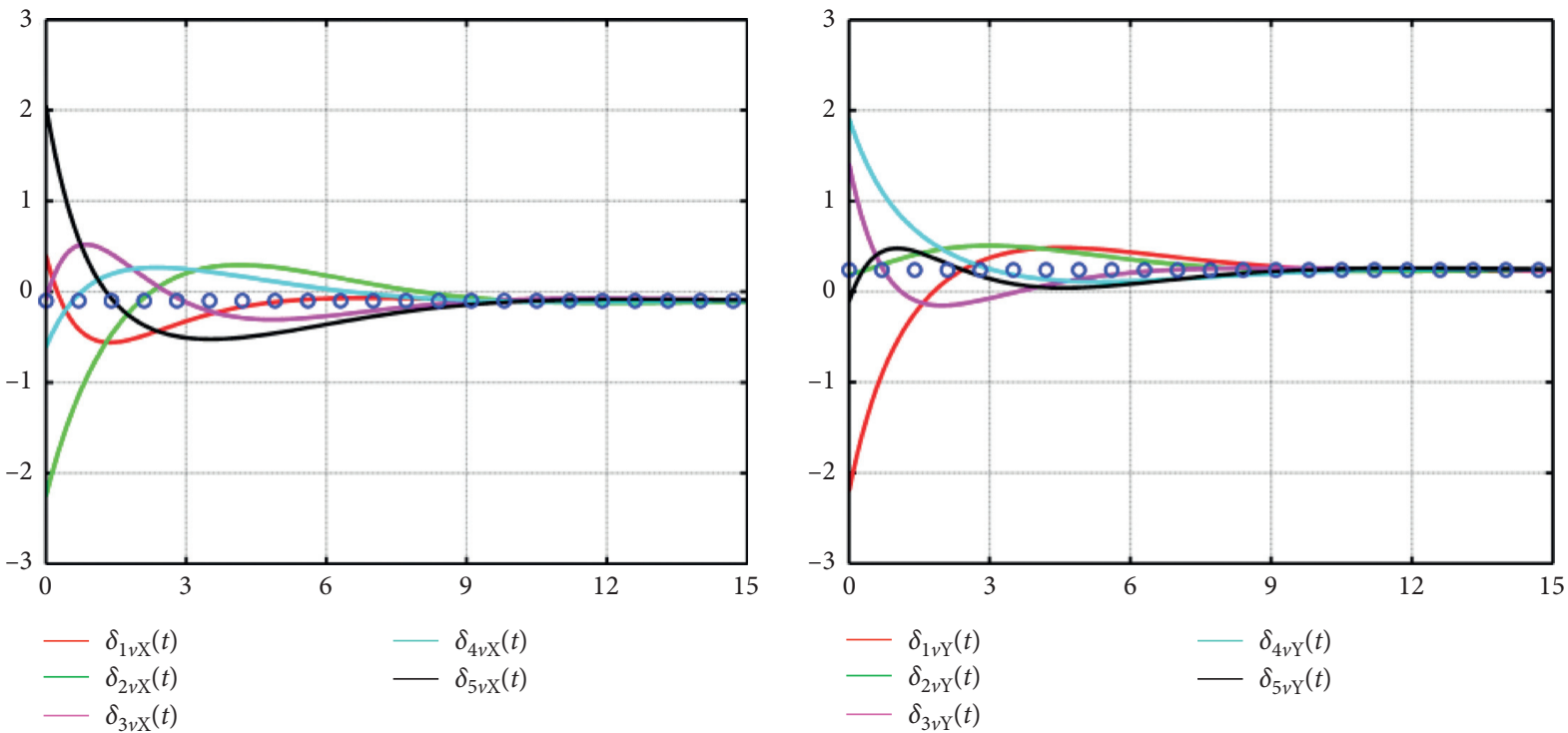

FIgURE 3: Trajectories of $\delta_{i v}(t)(i=1,2, \ldots, 5)$ in $X$ and $Y$ directions.

energy-limited formation control protocol (2) for leaderless MAS is achieved.

Example 2. (the leader-following case) In this example, the energy-limited multiagent system is composed of a leader and six followers, whose communication topology $G$ is shown in Figure 8. The dynamics of the followers are modeled by (35) with $x_{i}(t)=\left[x_{i X}(t), x_{i Y}(t), x_{i Z}(t)\right]^{T}$, $v_{i}(t)=\left[v_{i X}(t), v_{i Y}(t), v_{i Z}(t)\right]^{T}$ and $u_{i}(t)=\left[u_{i X}(t), u_{i Y}\right.$ $\left.(t), u_{i Z}(t)\right]^{T}(i \in\{2,3, \ldots, 7\})$. The followers need to keep a time-varying formation structure with the formation following function:

$$
\begin{aligned}
f_{i}(t)= & {\left[t \sin \left(\frac{(i-2) \pi}{3}+\frac{\pi}{2}\right), \frac{\sqrt{2}}{2} t \sin \left(\frac{(i-2) \pi}{3}\right), \frac{\sqrt{2}}{2} t \sin \left(\frac{(i-2) \pi}{3}\right),\right.} \\
& \left.\sin \left(\frac{(i-2) \pi}{3}+\frac{\pi}{2}\right), \frac{\sqrt{2}}{2} \sin \left(\frac{(i-2) \pi}{3}\right), \frac{\sqrt{2}}{2} \sin \left(\frac{(i-2) \pi}{3}\right)\right]^{T}, \quad(i \in\{2,3, \ldots, 7\}) .
\end{aligned}
$$




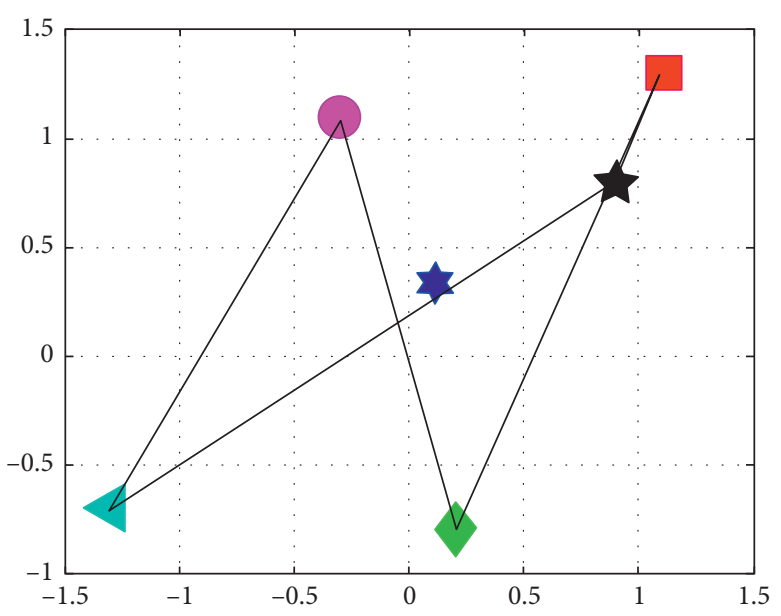

(a)

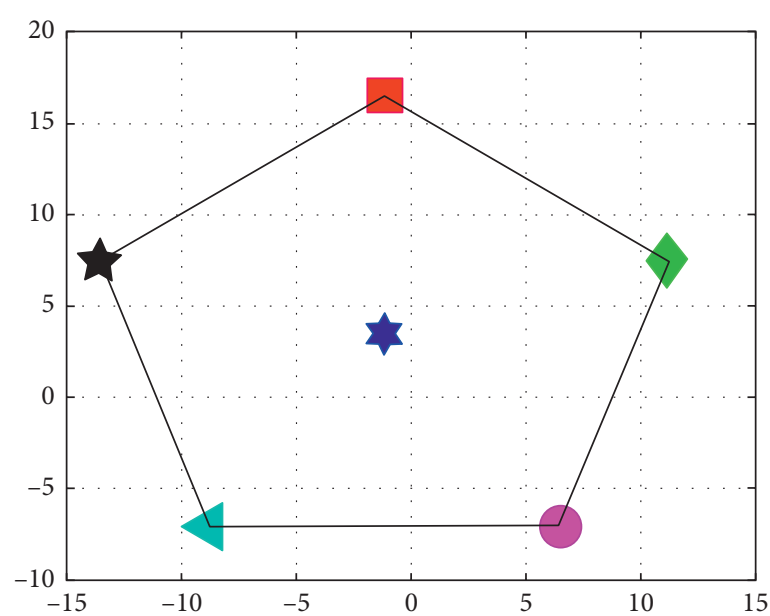

(c)

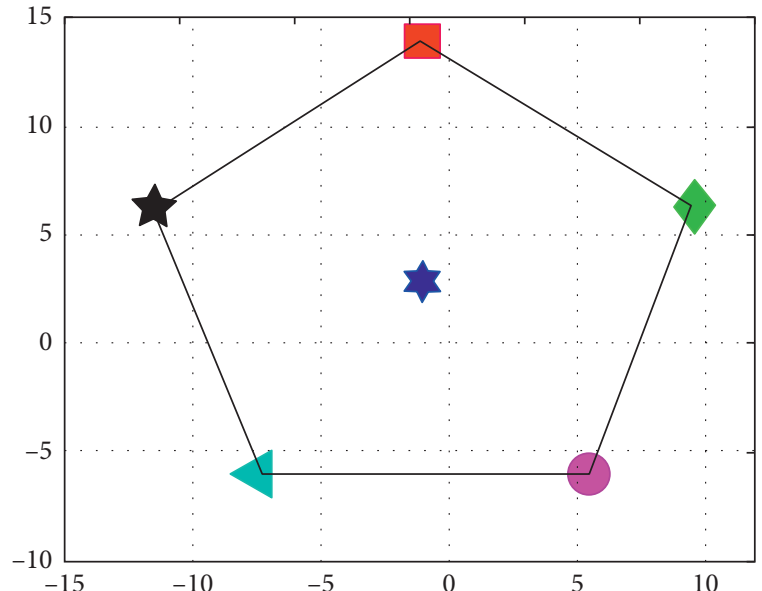

(b)

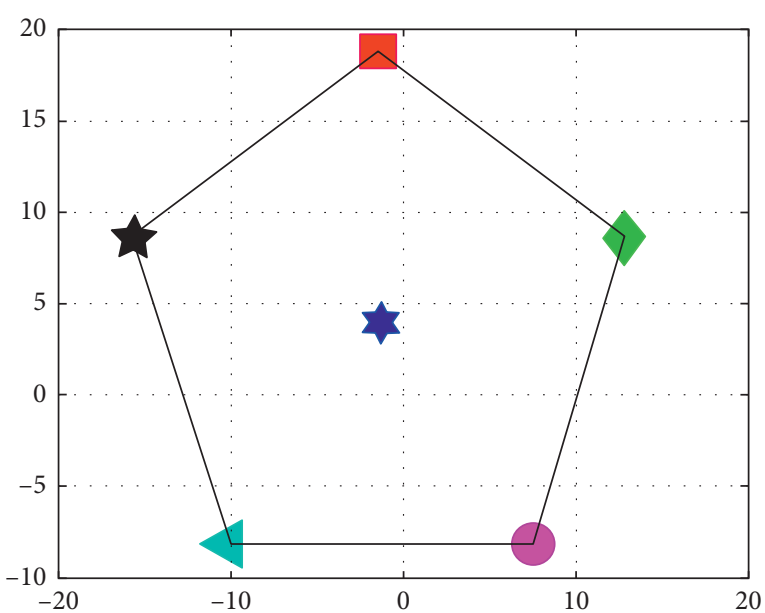

(d)

Figure 4: State snapshots of five agents at different times for the leaderless case. (a) $t=0 \mathrm{~s}$. (b) $t=12 \mathrm{~s}$. (c) $t=14 \mathrm{~s}$. (d) $t=15 \mathrm{~s}$.

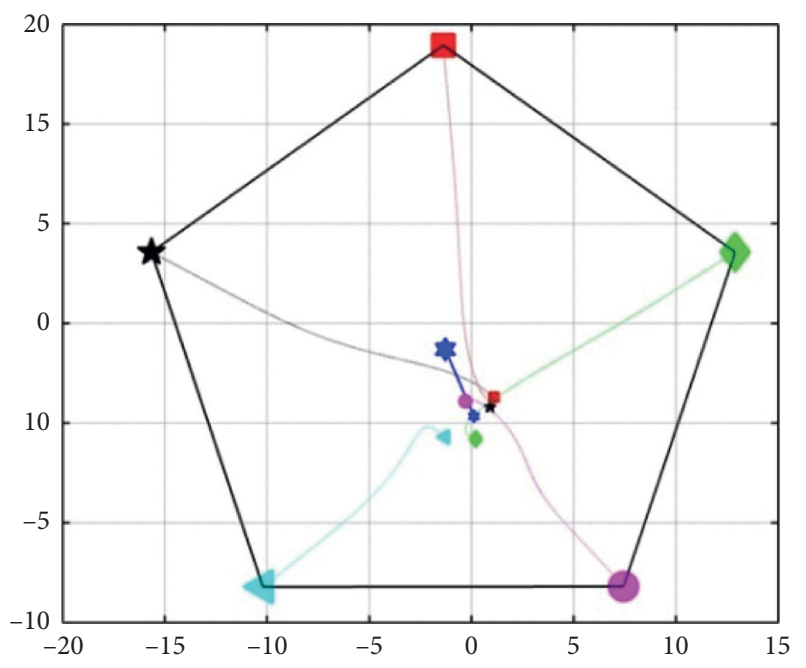

Figure 5: State trajectories of each agent for the leaderless case.

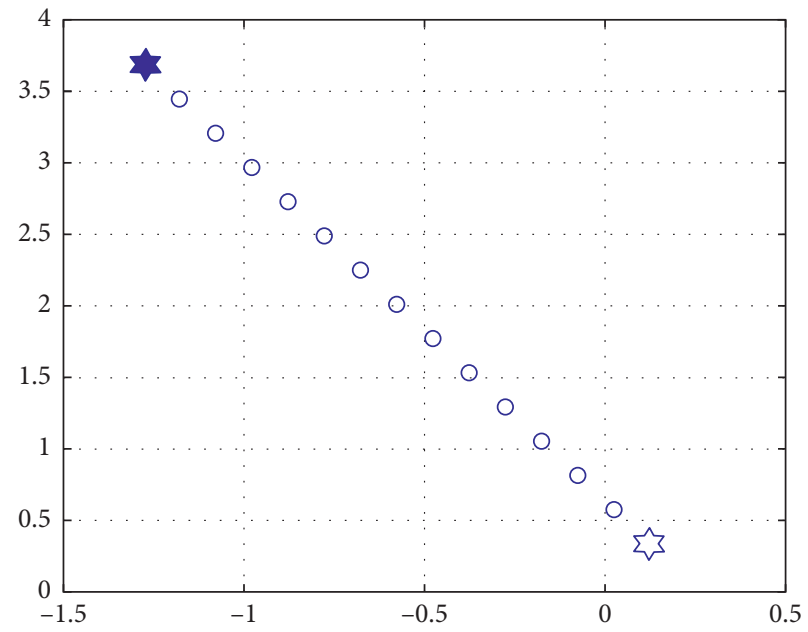

Figure 6: Trajectory of the formation center for the leaderless case. 


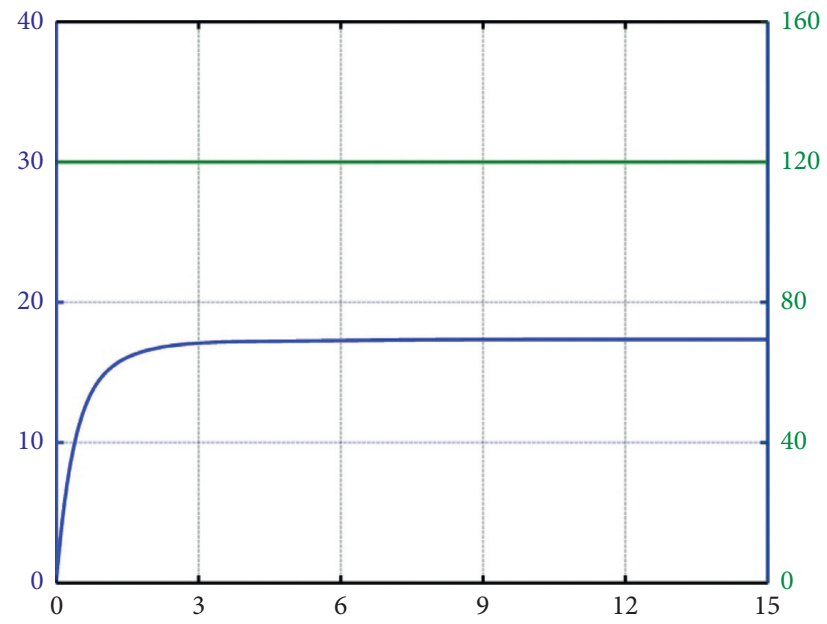

FIgURE 7: Trajectories of practical energy consumption and total energy supply for the leaderless case.

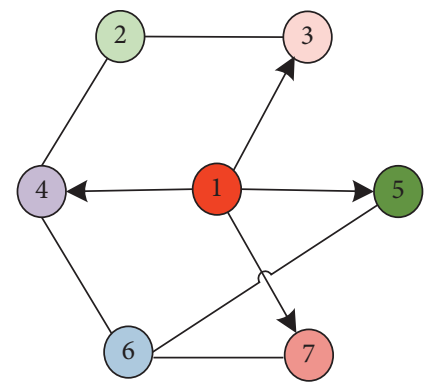

FIGURE 8: Communication topology $G$ for the leader-following case.
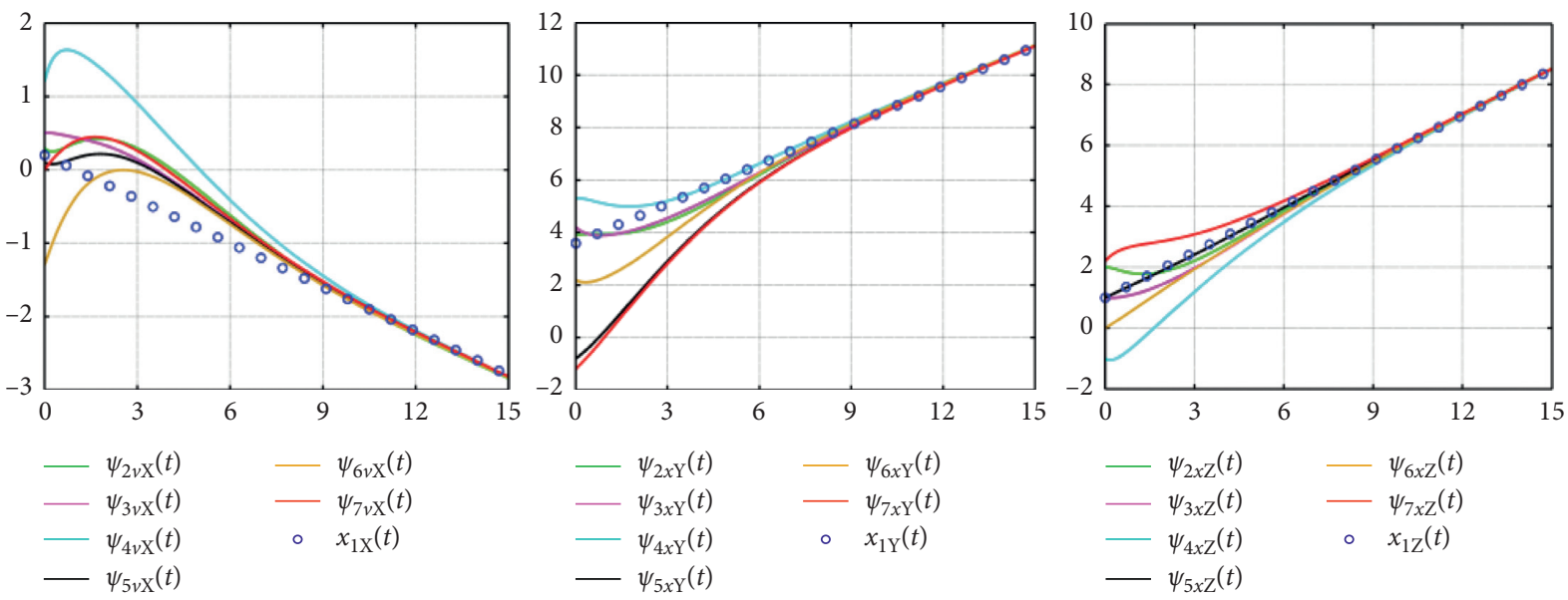

FIgure 9: Trajectories of $\psi_{i x}(t)(i=2,3, \ldots, 7)$ in $X, Y$, and $Z$ directions.

The initial states $\left[x_{i}(0), v_{i}(0)\right]^{T}=\left[x_{i X}(0), x_{i Y}(0), x_{i Z}(0)\right.$, $\left.v_{i X}(0), v_{i Y}(0), v_{i Z}(0)\right]^{T}(i \in\{1,2, \ldots, 7\})$ are as follows:

$$
\begin{aligned}
& {\left[x_{1}(0), v_{1}(0)\right]^{T}=[0.2,3.6,1.0,-0.2,0.5,0.5]^{T},} \\
& {\left[x_{2}(0), v_{2}(0)\right]^{T}=[0.3,3.9,2.0,0.5,0.2,0.0]^{T},} \\
& {\left[x_{3}(0), v_{3}(0)\right]^{T}=[0.5,4.2,1.0,0.6,-0.4,0.5]^{T},} \\
& {\left[x_{4}(0), v_{4}(0)\right]^{T}=[1.2,5.3,-1.0,1.3,0.8,0.0]^{T},} \\
& {\left[x_{5}(0), v_{5}(0)\right]^{T}=[0.1,-0.8,1.0,-1.2,0.9,0.5]^{T},} \\
& {\left[x_{6}(0), v_{6}(0)\right]^{T}=[-1.3,2.2,0.0,0.7,-1.3,0.0]^{T},} \\
& {\left[x_{7}(0), v_{7}(0)\right]^{T}=[0.0,-1.2,2.2,1.1,0.6,0.5]^{T} .}
\end{aligned}
$$

Choose $\kappa=0.12$ and the total energy $J_{u}^{*}=500$. According to Theorem 3, one can obtain that $0<k_{1}<0.3250$ and $0<k_{2}<$ 1.8045. In this case, we can choose $k_{1}=0.3$ and $k_{2}=1.0$.

Figures 9 and 10 show that state trajectories of $\psi_{i x}(t)$ and $\psi_{i v}(t)(i=2,3, \ldots, 7)$ for all followers along the $X$-axis, $Y$ axis, and $Z$-axis are asymptotically converge to the leader, respectively. In Figure 11, each follower is scattered in the three-dimensional plane by triangle, circle, parallelogram, hexagon, square, and pentagon at $t=0 \mathrm{~s}, t=12 \mathrm{~s}, t=14 \mathrm{~s}$, and $t=15 \mathrm{~s}$, where star denotes the leader. It can be seen 

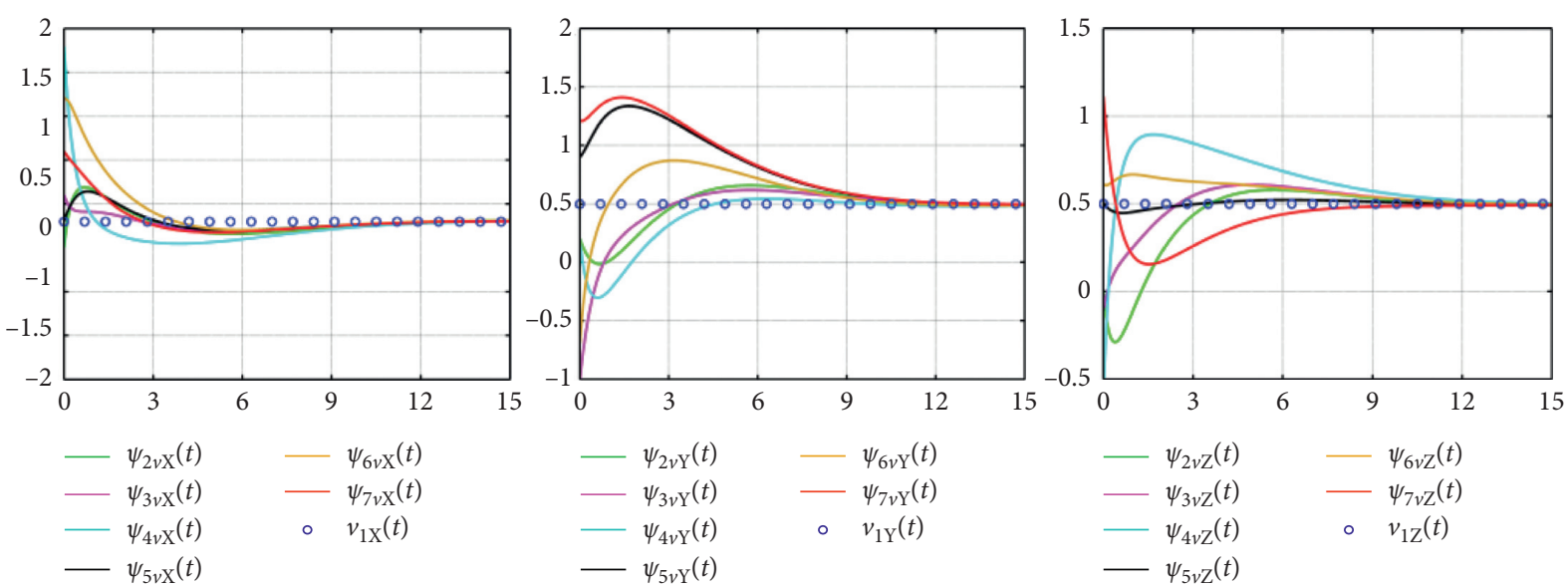

FIgURE 10: Trajectories of $\psi_{i v}(t)(i=2,3, \ldots, 7)$ in $X, Y$, and $Z$ directions.
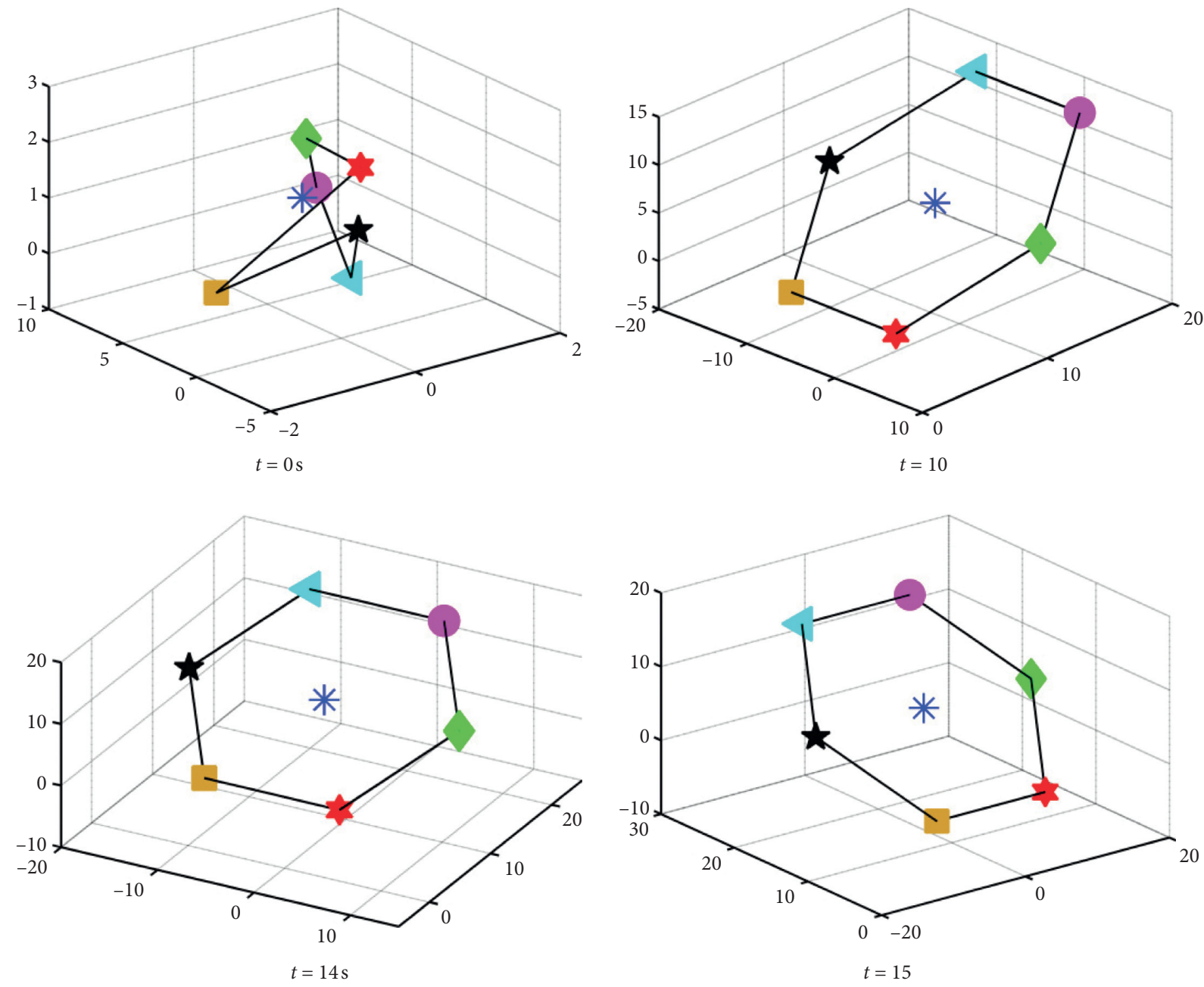

Figure 11: State snapshots of all agents at different times for the leader-following case. (a) $t=0 \mathrm{~s}$. (b) $t=12 \mathrm{~s}$. (c) $t=14 \mathrm{~s}$. (d) $t=15 \mathrm{~s}$.

that the followers finally form and move in the desired hexagonal formation structure around the leader. The state trajectory of the leader is shown in Figure 12. Compared with the leaderless simulation case, the biggest difference of the formation center function is the actual leader, but it is a virtual point for the leaderless case. From Figure 13, it can be noticed that the practical energy consumption $J_{u}$ converges to a finite value with $J_{u}<J_{u}^{*}$. From those simulation trajectories, it reveals that all followers can form a time-varying hexagonal structure formation to track the leader. 


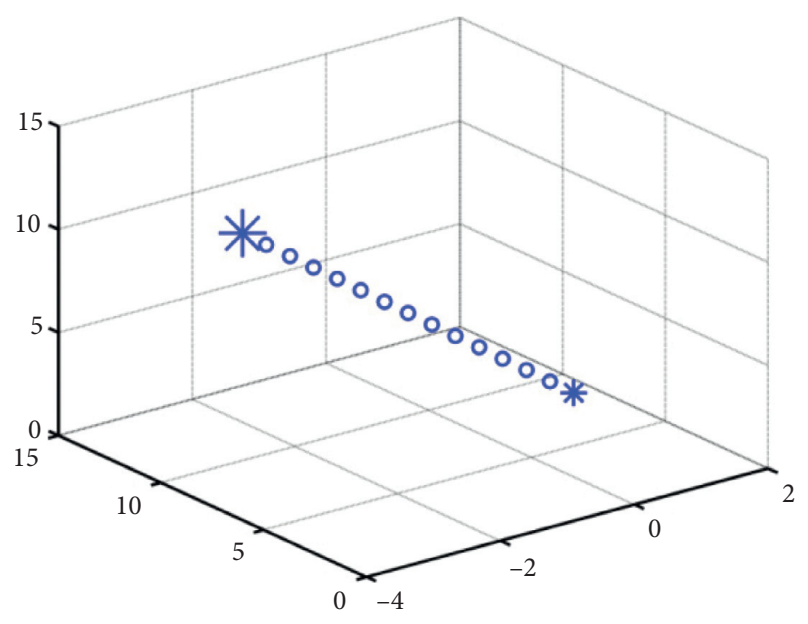

FIGURE 12: State trajectory of the leader for the leader-following case.

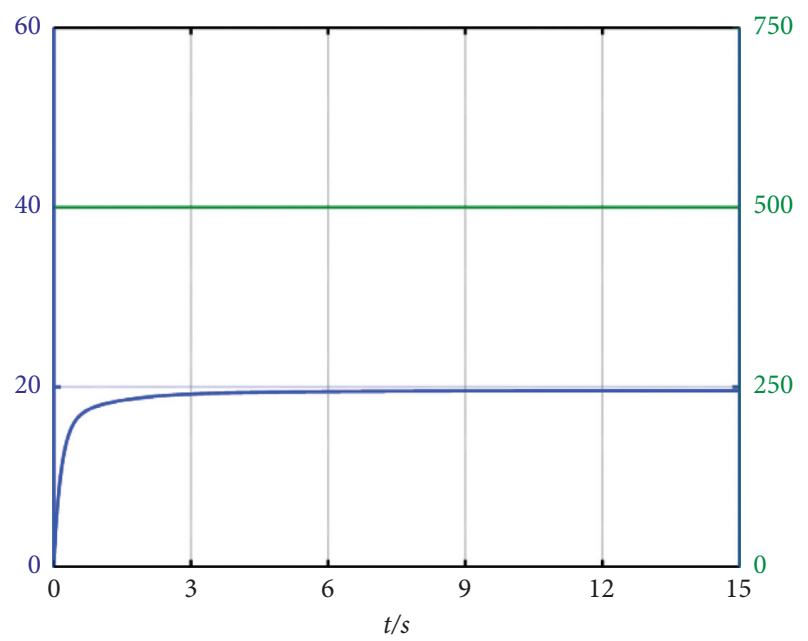

FIgURE 13: Trajectories of practical energy consumption and total energy supply for the leader-following case.

\section{Conclusions}

This paper investigates the ETVF control problem of secondorder MAS for leaderless and leader-following cases, respectively. In comparison with the existing results, the energy-limited time-varying protocol is firstly presented. A key idea of our method is the employment of energy constraint. Then, the stability is analyzed according to Lyapunov theory and graph theory, and the sufficient condition for secondorder MAS to achieve the time-varying formation is derived by using the joint design method of formation control gains and formation total energy supply. Meanwhile, the explicit formula of formation center function is used to describe the macroscopic movement of the whole multiagents. Moreover, we propose a two-step method to solve the problem of the asymmetric structure of the Laplacian matrix for the leader-following topology, which transforms the leaderfollowing one into the leaderless framework so that our work is simplified. In the future, research is aimed at extending the results to nonlinear MAS.

\section{Data Availability}

All data, such as the initial values and system dynamics, are provided in the simulation part of this paper and are verified by Matlab experiments.

\section{Conflicts of Interest}

The authors declare that they have no conflicts of interest.

\section{Authors' Contributions}

Yulong Zhao was responsible for validation and project administration. Wanzhen Quan contributed to conceptualization, methodology, and original draft preparation. Wanzhen Quan and Le Wang were responsible for investigation. Xiaogang Yang and Le Wang supervised the study. Xiaogang Yang was responsible for funding acquisition. All authors have read and agreed to the published version of the manuscript.

\section{Acknowledgments}

This study was supported by the National Natural Science Foundation of China under grant nos. 61867005, 61806209, 61763040 , and 61703411 and in part by the Open Foundation of Shaanxi Key Laboratory of Integrated and Intelligent Navigation under grant no. SKLIIN-20180103.

\section{References}

[1] F. Derakhshan and S. Yousefi, "A review on the applications of multiagent systems in wireless sensor networks," International Journal of Distributed Sensor Networks, vol. 15, no. 5, 2019.

[2] J. Qin, W. Fu, H. Gao, and W. X. Zheng, "Distributed k-means algorithm and fuzzy c-means algorithm for sensor networks based on multiagent consensus theory," IEEE Transactions on Cybernetics, vol. 47, no. 3, pp. 772-783, 2017.

[3] J. Qu, Z. Ji, C. Lin, and H. Yu, "Fast consensus seeking on networks with antagonistic interactions," Complexity, vol. 2018, Article ID 7831317, 15 pages, 2018.

[4] H. Liu, T. Ma, F. L. Lewis, and Y. Wan, "Robust formation control for multiple quadrotors with nonlinearities and disturbances," IEEE Transactions on Cybernetics, vol. 50, no. 4, pp. 1362-1371, 2020.

[5] J. Xi, L. Wang, J. Zheng, and X. Yang, "Energy-constraint formation for multiagent systems with switching interaction topologies," IEEE Transactions on Circuits and Systems I: Regular Papers, vol. 67, no. 7, pp. 2442-2454, 2020.

[6] J. Xi, C. Wang, X. Yang, and B. Yang, "Limited-budget output consensus for descriptor multiagent systems with energy constraints," IEEE Transactions on Cybernetics, vol. 50, no. 11, pp. 4585-4598, 2020.

[7] K.-K. Oh and H.-S. Ahn, "Formation control of mobile agents based on distributed position estimation," IEEE Transactions on Automatic Control, vol. 58, no. 3, pp. 737-742, 2013.

[8] S. Martin, "Multi-agent flocking under topological interactions," Systems \& Control Letters, vol. 69, pp. 53-61, 20149.

[9] G. Wen, Z. Duan, H. Su, G. Chen, and W. Yu, "A Connectivity-preserving flocking algorithm for multi-agent 
dynamical systems with bounded potential function," IET Control Theory \& Applications, vol. 6, no. 6, pp. 813-812, 2012.

[10] D. Yu, C. L. P. Chen, C.-E. Ren, and S. Sui, "Swarm control for self-organized system with fixed and switching topology," IEEE Transactions on Cybernetics, vol. 50, no. 10, p. 4481, 2020.

[11] M. Komareji, Y. Shang, and R. Bouffanais, "Consensus in topologically interacting swarms under communication constraints and time-delays," Nonlinear Dynamics, vol. 93, no. 3, pp. 1287-1300, 2018.

[12] Z. Ji, H. Lin, S. Cao, Q. Qi, and H. Ma, "The complexity in complete graphic characterizations of multiagent controllability," IEEE Transactions on Cybernetics, 2020, In press.

[13] L. Mo and S. Guo, "Consensus of linear multi-agent systems with persistent disturbances via distributed output feedback," Journal of Systems Science and Complexity, vol. 32, no. 3, pp. 835-845, 2019.

[14] S. Liu, Z. Ji, and H. Ma, "Jordan form-based algebraic conditions for controllability of multiagent systems under directed graphs," Complexity, vol. 2020, Article ID 7685460, 18 pages, 2020.

[15] X. Jia, L. Hu, F. Feng, and J. Xu, "Robust Ho consensus control for linear discrete-time swarm systems with parameter uncertainties and time-varying delays," International Journal of Aerospace Engineering, vol. 2019, Article ID 7278531, 16 pages, 2019.

[16] H. Zhao and J. H. Park, "Dynamic output feedback consensus of continuous-time networked multiagent systems," Complexity, vol. 20, no. 5, pp. 35-42, 2014.

[17] J. Xi, M. He, H. Liu, and J. Zheng, "Admissible output consensualization control for singular multi-agent systems with time delays," Journal of the Franklin Institute, vol. 353, no. 16, pp. 4074-4090, 2016.

[18] M. Ou, H. Du, and S. Li, "Finite-time formation control of multiple nonholonomic mobile robots," International Journal of Robust and Nonlinear Control, vol. 24, no. 1, pp. 140-165, 2014.

[19] T. Murayama, "Distributed model predictive consensus control for robotic swarm system," Artificial Life and Robotics, vol. 23, no. 4, pp. 628-635, 2018.

[20] J. Zhou and J. Yang, "Distributed guidance law design for cooperative simultaneous attacks with multiple missiles," Journal of Guidance, Control and Dynamics, vol. 39, no. 10, pp. 1-9, 2016.

[21] P. Dasgupta, "A multiagent swarming system for distributed automatic target recognition using unmanned aerial vehicles," IEEE Transactions on Systems, Man, and Cybernetics-Part A: Systems and Humans, vol. 38, no. 3, pp. 549-563, 2008.

[22] Z. Lin and H. Liu, "Topology-based distributed optimization for multi-UAV cooperative wildfire monitoring," Optimal Control Applications and Methods, vol. 38, no. 3, pp. 15301548, 2018.

[23] M. Fabris, A. Cenedese, and J. Hauser, "Optimal time-invariant formation tracking for a second-order multi-agent system," in Proceedings of the 2019 European Control Conference, pp. 1556-1561, Naples, Italy, June 2019.

[24] L. Dong, Y. Chen, X. Qu, and X. Qu, "Formation control strategy for nonholonomic intelligent vehicles based on virtual structure and consensus approach," Procedia Engineering, vol. 137, pp. 415-424, 2016.

[25] M. Li, Q. Ma, C. Zhou, J. Qin, and Y. Kang, "Distributed timevarying group formation control for generic linear systems with observer-based protocols," Neurocomputing, vol. 397, pp. 244-252, 2020.
[26] X. Dong, Y. Zhou, Z. Ren, and Y. Zhong, “Time-varying formation tracking for second-order multi-agent systems subjected to switching topologies with application to quadrotor formation flying," IEEE Transactions on Industrial Electronics, vol. 64, no. 6, pp. 5014-5024, 2017.

[27] H. Du, G. Wen, Y. Cheng, Y. He, and R. Jia, "Distributed finite-time cooperative control of multiple high-order nonholonomic mobile robots," IEEE Transactions on Neural Networks and Learning Systems, vol. 28, no. 12, pp. 29983006, 2016.

[28] Y. Jia, J. Du, W. Zhang, and L. Wang, "Three-Dimensional leaderless flocking control of large-scale small unmanned aerial vehicles," IFAC-PapersOnLine, vol. 50, no. 1 , pp. 6208-6213, 2017.

[29] Y. Li, C. Hua, and X. Guan, "Distributed output feedback leader-following control for high-order nonlinear multiagent system using dynamic gain method," IEEE Transactions on Cybernetics, vol. 50, no. 2, pp. 640-649, 2020.

[30] L. Gao, Y. Cui, W. Chen, and W. Chen, "Leader-following consensus for discrete-time descriptor multi-agent systems with observer-based protocols," Transactions of the Institute of Measurement and Control, vol. 38, no. 11, pp. 1353-1364, 2016.

[31] Z. Zhang, L. Zhang, F. Hao, and L. Wang, "Leader-following consensus for linear and Lipschitz nonlinear multiagent systems with quantized communication," IEEE Transactions on Cybernetics, vol. 47, no. 8, pp. 1970-1982, 2017.

[32] Z. Yan, X. Pan, Z. Yang, and L. Yue, "Formation control of leader-following multi-UUVs with uncertain factors and time-varying delays," IEEE Access, vol. 7, pp. 118792-118805, 2019.

[33] X. Dong, J. Xiang, L. Han, Q. Li, and Z. Ren, "Distributed time-varying formation tracking analysis and design for second-order multi-agent systems," Journal of Intelligent \& Robotic Systems, vol. 86, no. 2, pp. 277-289, 2016.

[34] X. Dong, Q. Li, Q. Zhao, and Z. Ren, "Time-varying group formation analysis and design for second-order multi-agent systems with directed topologies," Neurocomputing, vol. 205, pp. 367-374, 2016.

[35] L. Wang, J. Xi, M. He, and G. Liu, "Robust time-varying formation for multiagent systems with disturbances: extended-state-observer method," International Journal of Robust and Nonlinear Control, vol. 7, no. 30, pp. 2796-2808, 2020.

[36] Z.-H. Guan, B. Hu, M. Chi, D.-X. He, and X.-M. Cheng, "Guaranteed performance consensus in second-order multiagent systems with hybrid impulsive control," Automatica, vol. 50, no. 9, pp. 2415-2418, 2014.

[37] D. M. Zhang, L. Meng, X. G. Wang, and L. L. Ou, "Linear quadratic regulator control of multi-agent systems," Optimal Control Applications and Methods, vol. 36, no. 1, pp. 45-59, 2015.

[38] W. Dong, "Distributed optimal control of multiple systems," International Journal of Control, vol. 83, no. 10, pp. 20672079, 2010.

[39] C. Godsil and G. Royal, Algebraic Graph Theory, SpringerVerlag, New York, NY, USA, 2001. 